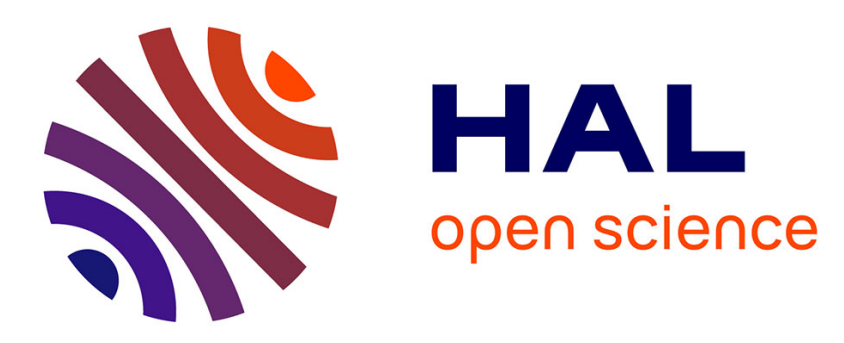

\title{
Using singular perturbations to reduce an epidemiological model: Application to Bovine Viral Diarrhoea Virus within-herd spread
}

Sébastien Gaucel, Béatrice Laroche, Pauline Ezanno, Elisabeta Vergu, Suzanne Touzeau

\section{To cite this version:}

Sébastien Gaucel, Béatrice Laroche, Pauline Ezanno, Elisabeta Vergu, Suzanne Touzeau. Using singular perturbations to reduce an epidemiological model: Application to Bovine Viral Diarrhoea Virus within-herd spread. Journal of Theoretical Biology, 2009, 258 (3), pp.426-436. 10.1016/j.jtbi.2008.08.011 . hal-00554504

\author{
HAL Id: hal-00554504 \\ https://hal.science/hal-00554504
}

Submitted on 11 Jan 2011

HAL is a multi-disciplinary open access archive for the deposit and dissemination of scientific research documents, whether they are published or not. The documents may come from teaching and research institutions in France or abroad, or from public or private research centers.
L'archive ouverte pluridisciplinaire HAL, est destinée au dépôt et à la diffusion de documents scientifiques de niveau recherche, publiés ou non, émanant des établissements d'enseignement et de recherche français ou étrangers, des laboratoires publics ou privés. 


\section{Author's Accepted Manuscript}

Using singular perturbations to reduce an epidemiological model: Application to Bovine Viral Diarrhoea Virus within-herd spread

Sébastien Gaucel, Béatrice Laroche, Pauline Ezanno, Elisabeta Vergu, Suzanne Touzeau

PII:

S0022-5193(08)00422-0

DOI: doi:10.1016/j.jtbi.2008.08.011

Reference: $\quad$ YJTBI 5256

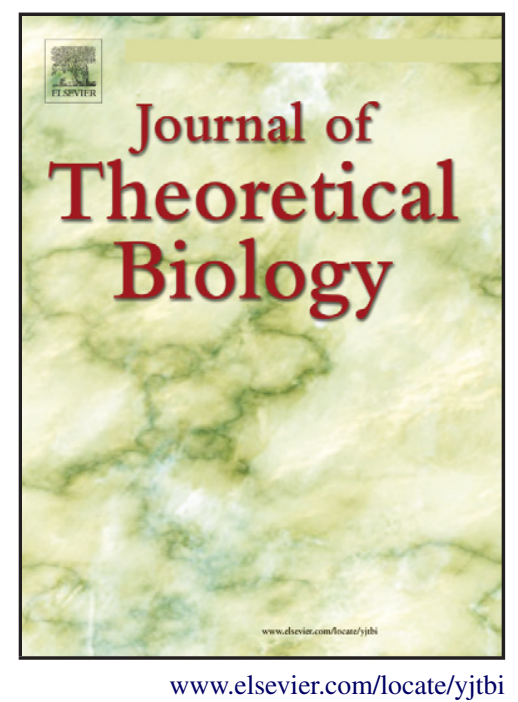

To appear in: Journal of Theoretical Biology

Received date: 13 February 2008

Revised date: 21 July 2008

Accepted date: $\quad 7$ August 2008

Cite this article as: Sébastien Gaucel, Béatrice Laroche, Pauline Ezanno, Elisabeta Vergu and Suzanne Touzeau, Using singular perturbations to reduce an epidemiological model: Application to Bovine Viral Diarrhoea Virus within-herd spread, Journal of Theoretical Biology (2008), doi:10.1016/j.jtbi.2008.08.011

This is a PDF file of an unedited manuscript that has been accepted for publication. As a service to our customers we are providing this early version of the manuscript. The manuscript will undergo copyediting, typesetting, and review of the resulting galley proof before it is published in its final citable form. Please note that during the production process errors may be discovered which could affect the content, and all legal disclaimers that apply to the journal pertain. 


\title{
Using singular perturbations to reduce an
} epidemiological model: application to Bovine

\section{Viral Diarrhoea Virus within-herd spread.}

Sébastien Gaucel ${ }^{\mathrm{a}, *, 1}$, Béatrice Laroche ${ }^{\mathrm{b}, \mathrm{c}, \mathrm{d}}$, Pauline Ezanno ${ }^{\mathrm{e}, \mathrm{f}}$, Elisabeta Vergu ${ }^{\text {a }}$, Suzanne Touzeau ${ }^{\text {a }}$

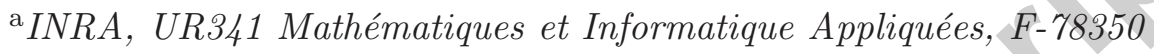

Jouy-en-Josas, France

b Univ. Paris-Sud, Laboratoire des Signaux et Systèmes, UMR8506, Gif-sur-Yvette, F-91190

${ }^{\mathrm{c}}$ CNRS, Gif-sur-Yvette, F-91190

d SUPELEC, Gif-sur-Yvette, F-91190

e INRA, UMR1300 Bio-agression, Epidémiologie et Analyse de Risque, F-4430־ Nantes, France

${ }^{\mathrm{f}}$ ENVN, UMR1300 Bio-agression, Epidémiologie, Analyse de Risque, F-4430־ Nantes, France

\begin{abstract}
Studying the spread of a pathogen in a managed metapopulation such as cattle herds in a geographical region often requires to take into account both the within- and between-herd transmission dynamics. This can lead to high-dimensional metapopulation systems resulting from the coupling of several within-herd transmission models. To tackle this problem, we aim in this
\end{abstract}


paper at reducing the dimension of a tractable but realistic dynamical system reproducing the within-herd spread. The context chosen to illustrate our purpose is Bovine Viral Diarrhoea Virus (BVDV) transmission in a cattle herd structured in two age classes and several epidemiological states, including two infectious states (transiently and persistently infected). Different time scales, corresponding to the epidemiological and demographic processes, are identified which allow to build a reduced model. Singular perturbation technique is used to prove that, under some non-restrictive conditions on parameter values, the behaviour of the original system is quite accurately approximated by that of the reduced system. Simulations are also performed to corroborate the approximation quality. Our study illustrates the methodological interest of using singular perturbations to reduce model complexity. It also rigorously proves the biologically intuitive assumption that transiently infected individuals can be neglected in a homogeneous population, when capturing the global dynamics of BVDV spread.

Key words: perturbation methods, time scales, model reduction, dynamical system, BVDV

* Corresponding author

Email addresses: Sebastien.Gaucel@grignon.inra.fr (Sébastien Gaucel),

Beatrice.Laroche@lss.supelec.fr (Béatrice Laroche), ezanno@vet-nantes.fr

(Pauline Ezanno), Elisabeta.Vergu@jouy.inra.fr (Elisabeta Vergu),

Suzanne.Touzeau@jouy.inra.fr (Suzanne Touzeau).

1 Current address: INRA, UMR1290 BIOGER CPP, BP 01, F-78850 ThivervalGrignon, France. 


\section{Introduction}

Theoretical studies on population dynamics most often involve relatively complex models, due to the intrinsic complexity of the phenomena that are captured (biological, demographic, etc.), to their interactions, and to the state dimension. Such is the case when studying the spread of a pathogen at a regional scale in a managed metapopulation such as cattle herds, which often requires to take into account the within-herd transmission dynamics, as well as the between-herd dynamics within the region of interest. Management induces an heterogeneous contact structure which needs to be taken into account for moderately propagating pathogens. This can lead to highdimensional metapopulation systems resulting from the coupling of several similar local within-herd transmission models. An appropriate approach to tackle this problem is to reduce the dimension of an epidemiological model designed to study the spread of a disease in a managed herd.

A possible solution for reducing the complexity consists in taking advantage of the presence of several time scales in the system. Indeed, events can intrinsically occur at different velocities which allows, under stability assumptions on the fast dynamics (see Khalil (1996) and appendix B), to neglect rapid events and therefore to build a lower-dimensional model, that can be handled analytically and that is governed by the dynamics of the slow events. Several mathematical methods allowing such system reduction, as aggregation of variables and singular perturbation, have been developed. These methods were used in biological fields, mainly in ecology (Lenbury, 1996; Lett et al., 2003), but also in epidemic modelling (González-Guzmán and Naulin, 1994; Song et al., 2002). From the methodological point of view, the aggregation 
of variables, perfect or approximate (Iwasa et al., 1987, 1989) are by far the most frequently used. The method, revised in Auger and Bravo de la Parra (2000), was first applied in the frame of ordinary differential equations (Auger and Roussarie, 1994; Auger and Poggiale, 1998) and then derived for partial differential equations (Auger and Roussarie, 1994; Bravo de la Parra et al., 2000), discrete dynamical systems (Bravo de la Parra et al., 1995; Sanz and Bravo de la Parra, 1999) or for stochastic frameworks (Sanz et al., 2003; Sanz and Bravo de la Parra, 2007). Several papers, studying dynamics of structured population models, have used the singular perturbation method (Arino et al., 1999; Song et al., 2002).

In this paper, we are interested in reducing the order of an epidemiological model designed to study the spread of a disease in a managed herd. We aim at using the dimensionally reduced form of the model for further studies in a metapopulation context. A reduced-order model is easier to manipulate and control and can therefore be coupled with other similar local models for studying the propagation at the between-herd scale. In order to achieve a trade-off between complexity and tractability, we chose to adapt a generic epidemiological model to a concrete situation: Bovine Viral Diarrhoea Virus (BVDV) epizootic dynamics within a managed herd through a simplified but realistic model.

BVDV virus is a pestivirus which does not survive in the environment. Hence, horizontal transmission mainly occurs by direct contacts with infected animals. It leads to transiently infected animals excreting low amounts of virus during a couple of weeks (Baker, 1987) before becoming immune. No clear and specific clinical signs are usually observed. However, if infection occurs during early or 
mid-pregnancy, it frequently generates embryonic and foetal deaths. For cows infected during mid-pregnancy that do not abort, vertical transmission is highly probable. These cows give birth to Persistently Infected (PI) calves (Hartley and Richards, 1988; Fray et al., 2000). PI animals excrete the virus during their whole life in a greater amount than transiently infected animals (Baker, 1987). PI dams always give birth to PI calves. PI animals have a higher lethality, with a half-life of one year (Houe, 1993). Consequently, the disease induces high economic losses, which is why several European countries are implementing control plans (Lindberg et al., 2006).

To model BVDV within-herd spread, a classical non structured SIR models (Susceptible / Infected / Recovered) is not adequate. Both vertical and horizontal transmissions should be considered as they lead to very different consequences. Transiently infected and PI animals cannot be regrouped in a single infectious status because their infectiousness and infection duration are drastically different. Hence, this herd structure should be considered when modelling BVDV spread. To date, several models have been developed to represent the spread of $\mathrm{BVDV}$ in a cattle herd; a review can be found in Viet et al. (2007). They generally aim at studying the effect of control measures and assessing efficiency. These models are usually complex models which integrate detailed herd dynamics and include most of current biological knowledge on BVDV transmission. Most often, this complexity renders their rigorous mathematical analysis impossible. Moreover, the metapopulation model generated by coupling several such models to study the between-herd transmission 
at a regional scale could be difficult to simulate. The model that we propose here, whilst simpler than such complex high-dimensional models, still preserves the main characteristics of BVDV spread. It takes into account the heterogeneities in transmission that require the introduction of different disease statuses. It represents a compromise between realistic complexity and mathematical tractability.

The paper is organised as follows: in section 2, we first present the BVDV model, then we analyse its structural properties. Exploiting the different time scales in the system corresponding to different biological processes, reducedorder models are built using the singular perturbation approach (Khalil, 1996); we then check the accuracy of these time scale approximations by using appropriate theorems (section 3). In order to illustrate our analytical results, simulations are presented using realistic parameter values (section 4). Finally we discuss the methodology and the biological interpretation of our findings (section 5).

\section{Epidemiological model}

We present in this section a model describing BVDV dynamics in a cattle herd located in a region where the disease in endemic. Hence, neighbouring herds contribute to the herd contamination. Purchases however are considered to be safe, as we assume that tests are performed for each animal introduced in the herd.

The mathematical model is first presented. This model exhibits different time scales between the demographic and epidemiological 
processes which are then outlined. Finally, we investigate the model equilibria.

\subsection{Model description}

The herd is structured in two age classes: Class 1, formed by juveniles (0-3 years), which are animals before breeding; and Class 2 formed by adults ( $>3$ years of age). This population structure is motivated by herd management, outflows, reproduction and entries in the herd depending on age.

In age class $i$, animals are classified into mutually exclusive BVDV health statuses (Fig. 1): susceptible $\left(S_{i}\right)$, transiently-infected $\left(T_{i}\right)$, persistently-infected $\left(P_{i}\right)$, recovered (i.e. immune; $\left.R_{i}\right)$. Recovered adults in age class 2 are actually divided into two statuses $\left(M_{2}\right.$ and $\left.R_{2}\right)$, because an infection during pregnancy can lead to embryonic or foetal death or also to the birth of a PI calf. Hence, status $M_{2}$ has been added to enable representing such specific consequences that may occur in the year following the infection. Adults in status $R_{2}$ are recovered adults that have calved at least once since infection or animals that have been infected as juveniles (coming from $R_{1}$ ).

Since two infectious statuses are considered (transiently-infected, status $T$, and persistently-infected, status $P$ ), two specific infection rates $\left(\beta_{T}\right.$ and $\beta_{P}$ respectively), independent of age are defined (Table 1). The force of infection in the herd - which drives the transition from the susceptible to the transientlyinfected status - is assumed to be density-dependent. Animals from both age classes are assumed to be equally in contact (homogeneous mixing in the 


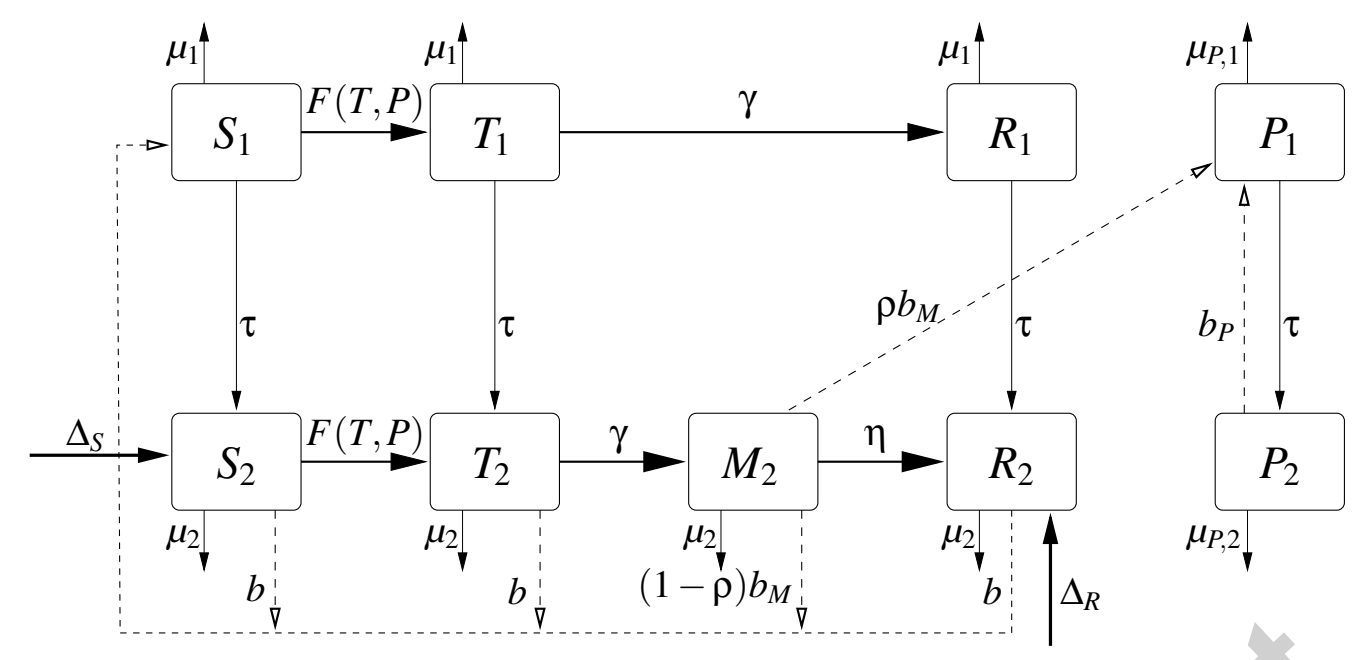

Fig. 1. Flow diagram of the epidemiological model representing BVDV health statuses per age classes and transitions between these states; in age class $i, S_{i}$ : susceptible, $T_{i}$ : transiently-infected, $P_{i}$ : persistently-infected, $R_{i}$ : recovered, $M_{2}$ : recovered but still pregnant since infection. Parameters are defined in Table 1 and the model equations are given by (1).

herd) and to have the same susceptibility to the disease. Hence, the force of infection will include $\beta_{T} T$ and $\beta_{P} P$ terms, with $T$ the total number of transiently-infected animals $\left(T_{1}+T_{2}\right)$ and $P$ the total number of persistentlyinfected animals $\left(P_{1}+P_{2}\right)$. We assume a constant additional infection rate $\beta_{v}$ due to neighbouring contacts with infected animals of other herds. Thus, the force of infection is defined by $F(T, P)=\beta_{T} T+\beta_{P} P+\beta_{v}$.

After a transient infection, animals are assumed to recover with a recovery rate $\gamma$, independent of age. Recovered juveniles go to status $R_{1}$. Recovered adults first go to status $M_{2}$, and then to status $R_{2}$ with rate $\eta$ (Table 1 ).

We assume that the infection has a strong impact on reproduction. Among adults in status $M_{2}$, some have been infected during pregnancy and thus can 
Table 1

Definition of the parameters used in the model.

\begin{tabular}{|c|c|c|}
\hline Parameters & Interpretation & Dimension* \\
\hline$b$ & Natural birth rate & $t^{-1}$ \\
\hline$b_{P}$ & Birth rate for PI infected individuals & $t^{-1}$ \\
\hline$b_{M}$ & Birth rate for individuals infected during pregnancy & $t^{-1}$ \\
\hline$\mu_{1}, \mu_{2}$ & $\begin{array}{l}\text { Age-dependent mortality rate for non PI individu- } \\
\text { als }\end{array}$ & \\
\hline$\mu_{P_{1}}, \mu_{P_{2}}$ & Age-dependent mortality rate for PI individuals & $t^{-1}$ \\
\hline$\tau$ & Maturating rate & $t^{-1}$ \\
\hline$\Delta_{S}, \Delta_{R}$ & $\begin{array}{l}\text { Purchases of susceptible or resistant mature indi- } \\
\text { viduals }\end{array}$ & $N / t$ \\
\hline$\beta_{T}$ & $\begin{array}{l}\text { Infection rate due to transiently infected individu- } \\
\text { als }\end{array}$ & $(N . t)^{-1}$ \\
\hline$\beta_{P}$ & Infection rate due to PI infected individuals & $(N . t)^{-1}$ \\
\hline$\beta_{v}$ & External infection rate & $t^{-1}$ \\
\hline$\gamma$ & Recovery rate & $t^{-1}$ \\
\hline$\rho$ & Vertical transmission ratio & - \\
\hline$\eta$ & $" M_{2} \rightarrow R_{2} "$ transfer rate & $t^{-1}$ \\
\hline
\end{tabular}

${ }^{*} t=$ time, $N=$ numbers 
abort. Hence, the birth rate in status $M_{2}\left(b_{M}\right)$ is assumed to be reduced compared to the natural birth rate applied to other statuses $\left(b_{S}=b_{T}=\right.$ $\left.b_{R}=b\right)$. A specific birth rate is also considered for persistently-infected adults (status $P_{2}$; rate $b_{P}$ ) to account for a potential impact of the disease on the reproduction of PI animals. A systematic vertical transmission is considered for pregnant PI adults (status $P_{2}$ ). A partial vertical transmission is considered for pregnant adults in status $M_{2}$, with rate $\rho$; the remaining calves are born in status $S_{1}$. Juveniles in all health statuses become adults with a maturating rate $\tau$.

Outflows in such a managed population consist in natural mortality, culling and selling. The resulting global so-called mortality rate depends on age $\left(\mu_{1}\right.$ and $\mu_{2}$ for juveniles and adults, respectively). These rates apply to all health statuses except status $P$. For status $P$, a disease-related mortality occurs in addition to these global mortality rates. Hence, a specific age-dependent mortality rate is defined ( $\mu_{P_{1}}$ and $\mu_{P_{2}}$ for PI juveniles and PI adults, respectively).

Finally, adult animals can be purchased and introduced in the herd. Such inflows are assumed to be perfectly secure, considering automatic detection and rejection of animals able to spread the infection in the herd. As a result, no entry of infected animals (statuses $T_{2}$ or $P_{2}$ ) or of immune dams carrying a PI foetus (status $M_{2}$ ) is allowed. Hence, inflows only occur in statuses $S_{2}$ and $R_{2}$, with constant entry flows $\Delta_{S}$ and $\Delta_{R}$, respectively. 
This leads to the following system of nine differential equations:

$$
\left\{\begin{array}{l}
\dot{S}_{1}=b\left(S_{2}+T_{2}+R_{2}\right)+(1-\rho) b_{M} M_{2}-\left(\tau+\mu_{1}\right) S_{1}-F(T, P) S_{1} \\
\dot{S}_{2}=\Delta_{S}+\tau S_{1}-\mu_{2} S_{2}-F(T, P) S_{2} \\
\dot{T}_{1}=-\left(\tau+\mu_{1}\right) T_{1}-\gamma T_{1}+F(T, P) S_{1} \\
\dot{T}_{2}=\tau T_{1}-\mu_{2} T_{2}-\gamma T_{2}+F(T, P) S_{2} \\
\dot{P}_{1}=b_{P} P_{2}+\rho b_{M} M_{2}-\left(\tau+\mu_{P_{1}}\right) P_{1} \\
\dot{P}_{2}=\tau P_{1}-\mu_{P_{2}} P_{2} \\
\dot{R}_{1}=-\left(\tau+\mu_{1}\right) R_{1}+\gamma T_{1} \\
\dot{M}_{2}=\gamma T_{2}-\left(\eta+\mu_{2}\right) M_{2} \\
\dot{R}_{2}=\Delta_{R}+\tau R_{1}+\eta M_{2}-\mu_{2} R_{2}
\end{array}\right.
$$

where: $T=T_{1}+T_{2}, \quad P=P_{1}+P_{2}, \quad F(T, P)=\beta_{T} T+\beta_{P} P+\beta_{v}$.

All parameters in this system are positive, as they represent positive quantities with biological meaning, and are summarised in Table 1.

We denote by $\mathbf{W}=\left(S_{1}, S_{2}, T_{1}, T_{2}, P_{1}, P_{2}, R_{1}, M_{2}, R_{2}\right)$ the state vector and by $\mathrm{W}(0)$ the initial condition. As these state variables represent population sizes, we set $\mathbf{W}(0) \geqslant 0$. The structure of the model then ensures that the state variables remain non negative in the course of time. Indeed, for system 1 the field is non negative along the boundaries of the non negative orthant. To prove this, let us consider that at time $t$, the $i$ th element of the state vector $w_{i}(t)=0$ and the other elements $w_{j \neq i} \geqslant 0(i, j \in\{1, \ldots, 9\})$. Straightforward computation shows that its derivative $\mathbf{w}_{\mathbf{i}}$, given by $(1)$, is then also non negative. This ensures that $w_{i}$ cannot become negative. So the non negative orthant is positively invariant. 


\subsection{Time scales}

The transient infection and recovery processes occur at a time scale that is faster than the demography. In other words, the corresponding epidemiological parameters are greater than the demographic parameters. We hence introduce a scaling parameter $\varepsilon$ such that:

$$
\beta_{T}=\frac{\beta_{T}^{\prime}}{\varepsilon}, \quad \beta_{P}=\frac{\beta_{P}^{\prime}}{\varepsilon}, \quad \gamma=\frac{\gamma^{\prime}}{\varepsilon}, \quad \text { with } 0<\varepsilon \ll 1
$$

Considering some typical population size $N_{m}$, we assume that $N_{m} \beta_{T}^{\prime}, N_{m} \beta_{P}^{\prime}$ are parameters of the same order of magnitude as the remaining model parameters. The contamination by neighbouring herds or the local environment is assumed to be relatively small compared to the contamination generated by within-herd contacts with infected animals. Hence, $\beta_{v}$ is smaller than the other epidemiological parameters and its order of magnitude is less than or equal to the order of magnitude of the demographic parameters. We finally assume that $\gamma^{\prime}$ has at least the same order of magnitude as the demographic parameter. In this case, system 1 is a two time scale system.

It is also reasonable to assume that individuals spend significantly more time in the susceptible state than in the transiently infected state, at least in moderately infected herds. It means that the recovery process runs faster than the infection process, in other words, that $\gamma^{\prime}$ has a greater order of magnitude than $N_{m} \beta_{T}^{\prime}, N_{m} \beta_{P}^{\prime}$, and the remaining model parameters. In that case, we introduce a second scaling parameter $\varepsilon^{\prime}$ such that:

$$
\gamma=\frac{\gamma^{\prime \prime}}{\varepsilon^{\prime}}, \quad \text { with } 0<\varepsilon^{\prime}<\varepsilon \ll 1
$$




\section{We hence obtain a three time scale system.}

All these assumptions on the order of magnitude of the parameters need to be verified on their numerical values to ensure relevant time scale separation in numerical simulations. This is done in section 4 .

\subsection{Equilibria}

Epidemiological models usually exhibit at least two equilibria, the trivial equilibrium, which corresponds to an extinct population, and a disease-free equilibrium. There may also be an endemic steady point. As described below, our system has only one endemic staedy state, provided that all parameters are positive.

In system (1), because of constant inflows corresponding to animal purchases, the population cannot become extinct and zero is not an equilibrium. Without these animal inflows (i.e. $\Delta_{S}=\Delta_{R}=0$ ) though, zero would be an equilibrium.

Moreover, due to the external contamination term which acts like an infection reservoir, there is no disease-free equilibrium in system (1). In the absence of this infection source (i.e. $\beta_{V}=0$ ), there would be such an equilibrium given by

$$
\overline{W^{0}}=\left(\overline{S_{1}^{0}}, \overline{S_{2}^{0}}, 0,0,0,0,0,0, \overline{R_{2}^{0}}\right),
$$

the non zero components of this vector being:

$$
\overline{S_{1}^{0}}=\frac{b\left(\Delta_{R}+\Delta_{S}\right)}{c_{S}}, \quad \overline{S_{2}^{0}}=\frac{b \tau \Delta_{R}+\mu_{2}\left(\mu_{1}+\tau\right) \Delta_{S}}{\mu_{2} c_{S}}, \quad \overline{R_{2}^{0}}=\frac{\Delta_{R}}{\mu_{2}},
$$

provided that the following parameter condition holds:

$$
c_{S}=\left(\mu_{1}+\tau\right) \mu_{2}-b \tau>0 .
$$


This condition corresponds to the existence and the stability of the disease-free equilibrium and ensures that the population does not explode.

For a non trivial set of parameters, we show that system (1) admits a non negative endemic steady state given by:

$$
\bar{W}=\left(\overline{S_{1}}, \overline{S_{2}}, \overline{T_{1}}, \overline{T_{2}}, \overline{P_{1}}, \overline{P_{2}}, \overline{R_{1}}, \overline{M_{2}}, \overline{R_{2}}\right), \quad \text { with } \bar{T}+\bar{P}>0 .
$$

$\bar{W}$ is a positive solution of $\dot{W}=0$. After certain algebraic manipulations, we show that solving this non linear system amounts to finding a positive root of a third order polynomial in $\overline{T_{1}}$ (or $\overline{T_{2}}$, both variables being quasi-symmetric), which has non analytically tractable formula. In order to circumvent this problem, we take advantage of the model time scales and use a second-order Taylor expansion of this polynomial around $\varepsilon$. For "small enough" values of $\varepsilon$ and provided that (4) and the following condition are verified:

$$
c_{P}=\left(\mu_{P_{1}}+\tau\right) \mu_{P_{2}}-b_{P} \tau>0
$$

we can then deduce the existence of a positive equilibrium $\bar{W}$ (see appendix A). Considering $\rho b_{M} M_{2}$ as an exogenous bounded input for the linear subsystem $\left(P_{1}, P_{2}\right)$, condition $(5)$ can be interpreted as the stability requirement ensuring the non explosion of the permanently infected population.

This equilibrium is proved to be exponentially stable (Khalil, 1996) in section 3.1 . 


\section{Model order reduction}

Thanks to the different time scales that occur in system $(1,2)$ and described in section 2.2, we can apply the singular perturbation theory to approximate this system by a lower-dimensional system. Theorems supporting our following analysis are given in appendix B.

\subsection{Two time scales}

With the following change of variables:

$$
A_{1}=S_{1}+T_{1}+R_{1}, \quad A_{2}=S_{2}+T_{2}+M_{2}
$$

system $(1,2)$ can be rewritten as:

$$
\left\{\begin{array}{l}
\dot{A}_{1}=b\left(A_{2}-M_{2}+R_{2}\right)+(1-\rho) b_{M} M_{2}-\left(\tau+\mu_{1}\right) A_{1}, \\
\dot{A}_{2}=\Delta_{S}+\tau\left(A_{1}-R_{1}\right)-\mu_{2} A_{2}-\eta M_{2}, \\
\dot{P}_{1}=b P_{2}+\rho b_{M} M_{2}-\left(\tau+\mu_{P_{1}}\right) P_{1}, \\
\dot{P}_{2}=\tau P_{1}-\mu_{P_{2}} P_{2}, \\
\dot{R_{2}}=\Delta_{R}+\tau R_{1}+\eta M_{2}-\mu_{2} R_{2}, \\
\varepsilon \dot{T}_{1}=-\varepsilon\left(\tau+\mu_{1}\right) T_{1}-\gamma^{\prime} T_{1}+\varepsilon F(T, P, \varepsilon)\left(A_{1}-T_{1}-R_{1}\right), \\
\varepsilon \dot{T}_{2}=\varepsilon \tau T_{1}-\varepsilon \mu_{2} T_{2}-\gamma^{\prime} T_{2}+\varepsilon F(T, P, \varepsilon)\left(A_{2}-T_{2}-M_{2}\right), \\
\varepsilon \dot{R}_{1}=-\varepsilon\left(\tau+\mu_{1}\right) R_{1}+\gamma^{\prime} T_{1}, \\
\varepsilon \dot{M}_{2}=\gamma^{\prime} T_{2}-\varepsilon\left(\eta+\mu_{2}\right) M_{2},
\end{array}\right.
$$

where: $T=T_{1}+T_{2}, P=P_{1}+P_{2}, \varepsilon F(T, P, \varepsilon)=\beta_{T}^{\prime} T+\beta_{P}^{\prime} P+\varepsilon \beta_{v}$. 
Setting $X=\left(A_{1}, A_{2}, P_{1}, P_{2}, R_{2}\right)$ and $Z=\left(T_{1}, T_{2}, R_{1}, M_{2}\right)$, this system takes the form of an autonomous singular perturbation model:

$$
\begin{aligned}
\dot{X} & =f(X, Z, \varepsilon), \\
\varepsilon \dot{Z} & =g(X, Z, \varepsilon) .
\end{aligned}
$$

Here $X$ and $Z$ are respectively called the slow and the fast variables.

The fast system (7b) has a unique non negative quasi steady state when $\varepsilon=0$ :

$$
Z^{*}=\left(T_{1}^{*}, T_{2}^{*}, R_{1}^{*}, M_{2}^{*}\right)=h(X)=\left(0,0, A_{1}, A_{2}\right) .
$$

By substituting this quasi steady state in the slow system (7a), we obtain the following linear reduced system:

$$
\left\{\begin{array}{l}
\dot{A}_{1}=(1-\rho) b_{M} A_{2}+b R_{2}-\left(\tau+\mu_{1}\right) A_{1}, \\
\dot{A}_{2}=\Delta_{S}-\left(\eta+\mu_{2}\right) A_{2}, \\
\dot{P}_{1}=\rho b_{M} A_{2}+b_{P} P_{2}-\left(\mu_{P_{1}}+\tau\right) P_{1}, \\
\dot{P}_{2}=\tau P_{1}-\mu_{P_{2}} P_{2}, \\
\dot{R}_{2}=\Delta_{R}+\tau A_{1}+\eta A_{2}-\mu_{2} R_{2},
\end{array}\right.
$$

which is represented in Fig. 2.

If conditions (4-5) are verified, it allows a unique non negative steady state:

$$
X^{*}=\left(A_{1}^{*}, A_{2}^{*}, P_{1}^{*}, P_{2}^{*}, R_{2}^{*}\right),
$$

with:

$$
\mid \begin{array}{ll}
A_{1}^{*}=\frac{(1-\rho) b_{M}}{\mu_{1}+\tau} A_{2}^{*}+\frac{b}{\mu_{1}+\tau} R_{2}^{*}, & P_{1}^{*}=\frac{\Delta_{S} \rho b_{M} \mu_{P_{2}}}{\left(\mu_{2}+\eta\right) c_{P}}, \\
A_{2}^{*}=\frac{\Delta_{S}}{\mu_{2}+\eta}, & P_{2}^{*}=\frac{\Delta_{S} \rho b_{M} \tau}{\left(\mu_{2}+\eta\right) c_{P}}, \\
R_{2}^{*}=\frac{\mu_{1}+\tau}{c_{S}}\left(\Delta_{R}+\frac{\Delta_{S}}{\mu_{2}+\eta}\left(\eta+\frac{(1-\rho) b_{M} \tau}{\mu_{1}+\tau}\right)\right) .
\end{array}
$$




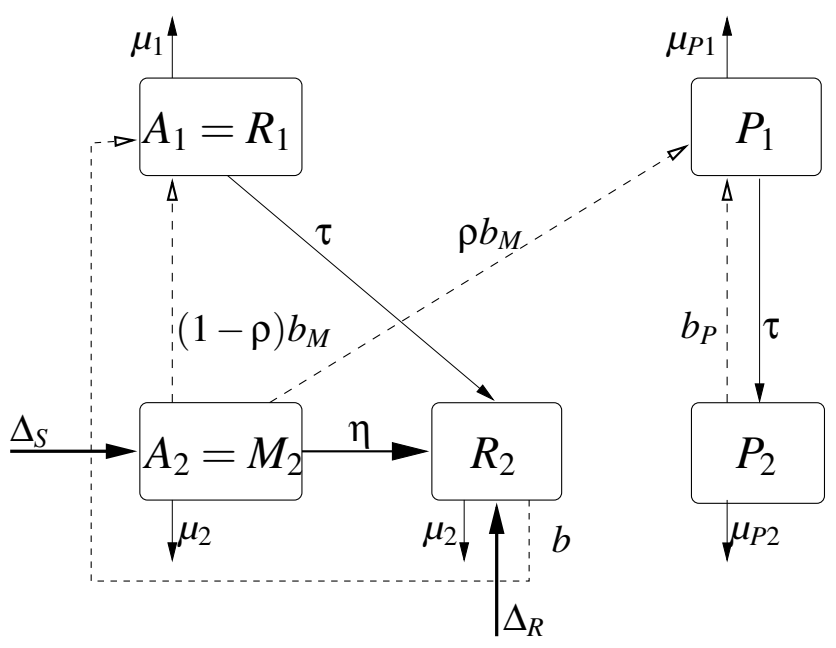

Fig. 2. Flow diagram of the reduced model given by equations (9), representing infection states per age classes and transitions between these states; recovered juveniles: $A_{1}=S_{1}+T_{1}+R_{1}=R_{1}$, recovered adults still in pregnancy since infection: $A_{2}=S_{2}+T_{2}+M_{2}=M_{2}$, recovered adults: $R_{2}$, persistently-infected juveniles and adults $R_{1}, R_{2}$. Parameters are defined in Table 1 .

After certain algebraic manipulations, we obtain the characteristic polynomial of the Jacobian matrix of system (9) in $X^{*}$ :

$\chi(\lambda)=-\left(\lambda+\mu_{1}+\tau\right)\left[\lambda^{2}+\left(\mu_{1}+\mu_{2}+\tau\right) \lambda+c_{S}\right]\left[\lambda^{2}+\left(\mu_{P_{1}}+\mu_{P_{2}}+\tau\right) \lambda+c_{P}\right]$.

Since $c_{S}$ and $c_{P}$ are positive, $-\chi$ is a product of first and second order polynomials, with real positive coefficients. According to the Routh-Hurwitz criterion, its roots, corresponding to the eigenvalues of system (9), are negative. As the reduced system is linear, $X^{*}$ is hence globally exponentially stable.

The boundary layer system verified by

$$
y=\left(t_{1}, t_{2}, r_{1}, r_{2}\right)=Z-h(X)=\left(T_{1}, T_{2}, R_{1}-A_{1}, M_{2}-A_{2}\right)
$$


with $d t=\varepsilon d \nu$ is:

$$
\left\{\begin{array}{l}
\frac{d t_{1}}{d \nu}=-\gamma^{\prime} t_{1}-\left(\beta_{T}^{\prime}\left(t_{1}+t_{2}\right)+\beta_{P}^{\prime} P\right)\left(t_{1}+r_{1}\right) \\
\frac{d t_{2}}{d \nu}=-\gamma^{\prime} t_{2}-\left(\beta_{T}^{\prime}\left(t_{1}+t_{2}\right)+\beta_{P}^{\prime} P\right)\left(t_{2}+m_{2}\right) \\
\frac{d r_{1}}{d \nu}=\gamma^{\prime} t_{1} \\
\frac{d r_{2}}{d \nu}=\gamma^{\prime} t_{2}
\end{array}\right.
$$

From the model structure, we deduce that $t_{i}(i=1,2)$ remain non negative and that $\left(t_{i}+r_{i}\right)(i=1,2)$ remain non positive. Hence, integrating and bounding $\frac{d\left(t_{i}+r_{i}\right)}{d \nu}$, we obtain:

$$
\left|\left(t_{i}(\nu)+r_{i}(\nu)\right)\right| \leqslant\left|\left(t_{i}(0)+r_{i}(0)\right)\right| e^{-\beta_{P} P \nu} .
$$

The last two equations of system (10) lead to:

$$
\frac{d v_{i}}{d \nu}=e^{\gamma^{\prime} \nu}\left(\frac{d r_{i}}{d \nu}+\gamma^{\prime} r_{i}\right)=\gamma^{\prime} e^{\gamma^{\prime} \nu}\left(t_{i}+r_{i}\right), \quad \text { with } v_{i}=e^{\gamma^{\prime} \nu} r_{i}
$$

Integrating and bounding this equation using (11), we obtain:

$$
\begin{gathered}
r_{i}(\nu)=r_{i}(0) e^{-\gamma^{\prime} \nu}+\gamma^{\prime} e^{-\gamma^{\prime} \nu} \int_{0}^{\nu} e^{\gamma^{\prime} \xi}\left(t_{i}+r_{i}\right) d \xi \\
\left|r_{i}(\nu)\right| \leqslant\left|r_{i}(0)\right| e^{-\gamma^{\prime} \nu}+\left|t_{i}(0)+r_{i}(0)\right| \frac{\gamma^{\prime}}{\gamma^{\prime}-\beta_{P}^{\prime} P}\left(e^{-\beta_{P}^{\prime} P \nu}-e^{-\gamma^{\prime} \nu}\right) .
\end{gathered}
$$

So the zero equilibrium of the boundary layer system (10) is globally exponentially stable.

Moreover $f, g, h$, the Jacobian of the reduced model $(9)$ and $\partial g(X, Z, 0) / \partial Z$ are smooth functions. Hence, by applying theorems 1 and 2 given in appendix B, we conclude that when $\varepsilon$ is "small enough":

- (from theorem 1) the reduced model is a good approximation of the original system (1), with $Z=Z^{*}=h(X)$ given by (8); 
- (from theorem 2) the endemic steady state of system (1), obtained when conditions (4-5) are verified, is globally exponentially stable.

\subsection{Three time scales}

In order to reduce the three time scale system $(1,3)$, we will proceed in two steps.

(1) First, we choose the time scale of recovery as fast scale, and we pool demography and infection time scales into the slow time scale, applying the singular perturbation theory in the same way as in the previous section.

(2) In a second step, we consider the reduced system obtained in step 1 as the new original system.

As in previous section, we need a change of variables to rewrite the original system in the singular perturbation form. Taking as new variables

$$
A_{1}=S_{1}+T_{1}+R_{1}, \quad A_{2}=S_{2}+T_{2}+M_{2}, \quad G_{1}=T_{1}+R_{1}, \quad G_{2}=T_{2}+M_{2}
$$


system $(1,3)$ becomes:

$$
\left\{\begin{array}{l}
\dot{A}_{1}=b\left(A_{2}-M_{2}+R_{2}\right)+(1-\rho) b_{M} M_{2}-\left(\tau+\mu_{1}\right) A_{1}, \\
\dot{A}_{2}=\Delta_{S}+\tau\left(A_{1}-R_{1}\right)-\mu_{2} A_{2}-\eta M_{2}, \\
\dot{P}_{1}=b P_{2}+\rho b_{M} M_{2}-\left(\tau+\mu_{P_{1}}\right) P_{1}, \\
\dot{P}_{2}=\tau P_{1}-\mu_{P_{2}} P_{2}, \\
\dot{R}_{2}=\Delta_{R}+\tau R_{1}+\eta M_{2}-\mu_{2} R_{2}, \\
\dot{G}_{1}=-\left(\tau+\mu_{1}\right) G_{1}+F(G, P, \varepsilon)\left(A_{1}-G_{1}\right), \\
\dot{G}_{2}=\tau\left(G_{1}-R_{1}\right)-\mu_{2} G_{2}-\eta M_{2}+\varepsilon F(G, P, \varepsilon)\left(A_{2}-G_{2}\right), \\
\varepsilon^{\prime} \dot{R}_{1}=-\varepsilon^{\prime}\left(\tau+\mu_{1}\right) R_{1}+\gamma^{\prime \prime}\left(G_{1}-R_{1}\right), \\
\varepsilon^{\prime} \dot{M}_{2}=\gamma^{\prime \prime}\left(G_{2}-M_{2}\right)-\varepsilon^{\prime}\left(\eta+\mu_{2}\right) M_{2},
\end{array}\right.
$$

where: $G=G_{1}+G_{2}, P=P_{1}+P_{2}$,

$$
\varepsilon F(G, P, \varepsilon)=\beta_{T}^{\prime}\left(G-\left(R_{1}+M_{2}\right)\right)+\beta_{P}^{\prime} P+\varepsilon \beta_{v} .
$$

We obtain respectively slow and fast variables:

$$
X=\left(A_{1}, A_{2}, P_{1}, P_{2}, R_{2}, G_{1}, G_{2}\right) \text { and } Z=\left(R_{1}, M_{2}\right) \text {. }
$$

The fast system of (12) has a unique non negative quasi steady state when $\varepsilon^{\prime}=0$

$$
\widetilde{Z}=\left(\widetilde{R_{1}}, \widetilde{M_{2}}\right)=h(X)=\left(G_{1}, G_{2}\right)
$$


Substituting this quasi steady state in the slow system, we obtain the following semi-reduced system:

$$
\left\{\begin{array}{l}
\dot{A}_{1}=b\left(A_{2}+R_{2}\right)+\left((1-\rho) b_{M}-b\right) G_{2}-\left(\tau+\mu_{1}\right) A_{1}, \\
\dot{A}_{2}=\Delta_{S}+\tau\left(A_{1}-G_{1}\right)-\mu_{2} A_{2}-\eta G_{2}, \\
\dot{P}_{1}=b P_{2}+\rho b_{M} G_{2}-\left(\tau+\mu_{P_{1}}\right) P_{1}, \\
\dot{P}_{2}=\tau P_{1}-\mu_{P_{2}} P_{2}, \\
\dot{R}_{2}=\Delta_{R}+\tau G_{1}+\eta G_{2}-\mu_{2} R_{2}, \\
\dot{G}_{1}=-\left(\tau+\mu_{1}\right) G_{1}+\left(\frac{\beta_{P}^{\prime}}{\varepsilon} P+\beta_{v}\right)\left(A_{1}-G_{1}\right), \\
\dot{G}_{2}=-\left(\mu_{2}+\eta\right) G_{2}+\left(\frac{\beta_{P}^{\prime}}{\varepsilon} P+\beta_{v}\right)\left(A_{2}-G_{2}\right) .
\end{array}\right.
$$

If conditions (4-5) are verified, and for $\varepsilon$ sufficiently small, semi-reduced system (14) has a unique non negative steady state $\widetilde{X}$ (see appendix A for details).

$$
\widetilde{X}=\left(\widetilde{A_{1}}, \widetilde{A_{2}}, \widetilde{P_{1}}, \widetilde{P_{2}}, \widetilde{R_{2}}, \widetilde{G_{1}}, \widetilde{G_{2}}\right)
$$

Let us consider system (14) as our new original system, which is already in singular perturbation form with $\mathbf{X}=\left(A_{1}, A_{2}, P_{1}, P_{2}, R_{2}\right)$ and $\mathbf{Z}=\left(G_{1}, G_{2}\right)$ as fast and slow variables. The fast system of (14) has a unique non negative quasi steady state when $\varepsilon=0$ given by

$$
\mathbf{Z}^{*}=\left(G_{1}^{*}, G_{2}^{*}\right)=\mathbf{h}(\mathbf{X})=\left(A_{1}, A_{2}\right)
$$

Substituting this quasi steady state in the slow part of system (14), we obtain system (9) as reduced system. We saw in section 3.1 that the steady state of (9) is exponentially stable. Setting $\mathbf{y}=\left(\mathbf{y}_{\mathbf{1}}, \mathbf{y}_{\mathbf{2}}\right)=\mathbf{Z}-h(X)=\left(G_{1}-A_{1}, G_{2}-A_{2}\right)$ 
and $d t=\varepsilon d \nu$, we write the boundary layer system as:

$$
\left\{\begin{array}{l}
\frac{d \mathbf{y}_{\mathbf{1}}}{d \nu}=-\beta_{P}^{\prime} P \mathbf{y}_{\mathbf{1}} \\
\frac{d \mathbf{y}_{\mathbf{2}}}{d \nu}=-\beta_{P}^{\prime} P \mathbf{y}_{\mathbf{2}}
\end{array}\right.
$$

As system (15) is linear with negative eigenvalues, it is exponentiallly stable, uniformly in $(t, \mathbf{X})$. By applying theorem 1 , we conclude that, for $\varepsilon$ "small enough", the reduced system (9) is a good approximation of semireduced system (14). In addition, with the appropriate shift of the origin, hypotheses of theorem 2 are also verified. So we obtain the exponential stability of $\widetilde{X}$ for the semi-reduced system (14).

Let us now consider system (12) and its approximation given by semi-reduced system (14). The corresponding boundary layer system verified by:

$$
y=\left(y_{1}, y_{2}\right)=Z-h(X)=\left(R_{1}-G_{1}, M_{2}-G_{2}\right)
$$

with $d t=\varepsilon^{\prime} d \nu$ is:

$$
\left\{\begin{array}{l}
\frac{d y_{1}}{d \nu}=-\gamma^{\prime \prime} y_{1} \\
\frac{d y_{2}}{d \nu}=-\gamma^{\prime \prime} y_{2}
\end{array}\right.
$$

As (16) is linear and independent of $(t, X)$, the origin is trivially exponentially stable uniformly in $(t, X)$. Hence, theorem 1 allows to conclude that, for $\varepsilon^{\prime}$ "small enough", the reduced model (14) is a good approximation of the complete system $(1,3)$.

Finally, as in previous section, we conclude that, for well chosen $\varepsilon$ and $\varepsilon^{\prime}$, the reduced model (9) is a good approximation of the complete system $(1,3)$. 


\section{Simulations}

Simulations were performed using the Scilab $^{2}$ standard ODE integration procedure. We used realistic parameters values which are summarised in Table 2. Most parameter values were taken from the literature. When such referenced values were not available, we considered reasonable assumptions as follows: we assume that infection has no effect on the fertility of PI animals, so we set $b_{P}=b ; \beta_{v}$ was adjusted in order to obtain prevalences in the herd consistent with observations in BVDV endemic regions. Numerical values provided in published studies are not always errorless since biological data are often incomplete or scarce and sometimes missing. From this point of view, we have in our model two kinds of parameters: (i) well known parameters, namely demographic parameters $\left(b, \tau, \mu_{1}\right.$ and $\left.\mu_{2}\right)$ and parameters related to purchases $\left(\Delta_{S}\right.$ and $\left.\Delta_{R}\right)$, which are typical for cattle herds; (ii) parameters which are less well characterised, such as transmission parameters and those modelling the disease epidemiology $\left(\beta_{T}, \beta_{P}, \rho, \gamma, \eta\right)$ or its direct impact on animal health $\left(\mu_{P 1}\right.$ and $\left.\mu_{P 2}\right)$. For the second group of parameters, sensible values corresponding to average patterns (when several different values were available in the literature) were derived from Ezanno et al. (2007), where these values are supported by a sensitivity analysis. Since time is measured in years in our simulations, the parameter units were set accordingly.

$\overline{2 \text { Scilab }}$, a free scientific software for numerical computation: http://www. scilab. org 
Table 2

Realistic parameter values used for the numerical simulations (unit); parameter definitions are given in Table 1.

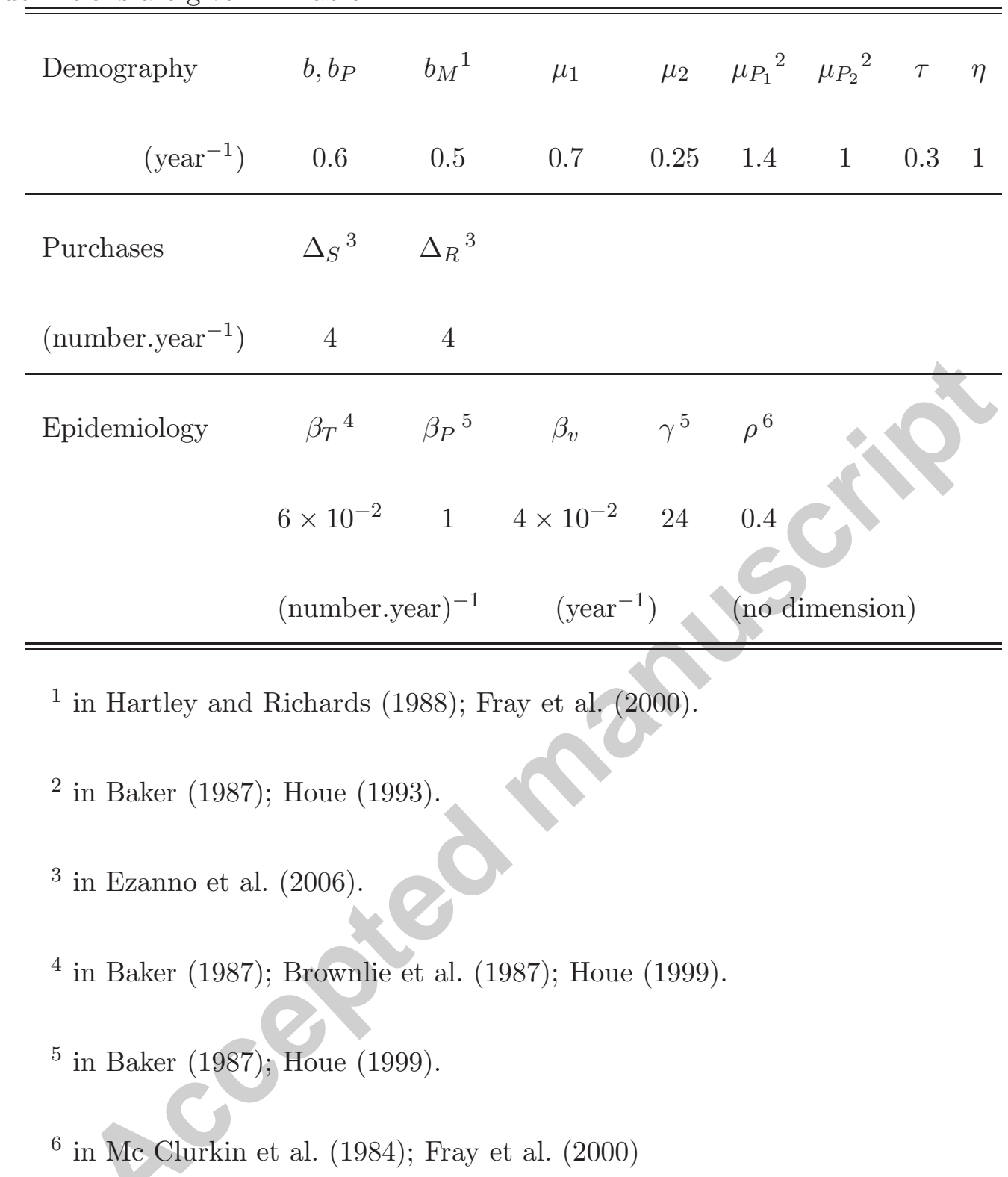

For these parameter values, existence conditions $(4,5)$ are fulfilled so there is an endemic steady state. Numerical computation of this equilibrium state for 
the complete system leads to the following values:

$$
\begin{gathered}
\overline{S_{1}}=25.5, \quad \overline{S_{2}}=7, \quad \overline{P_{1}}=1, \quad \overline{P_{2}}=0.3, \quad \overline{R_{2}}=96, \\
\overline{T_{1}}=1.5, \quad \overline{T_{2}}=0.5, \quad \overline{R_{1}}=35, \quad \overline{M_{2}}=8.5, \\
\left(\overline{A_{1}}=62, \quad \overline{A_{2}}=16, \quad \overline{G_{1}}=36.5, \quad \overline{G_{2}}=9\right) .
\end{gathered}
$$

All simulations are performed using the following initial state expressed in numbers:

$$
\mathbf{W}(0)=(65,110,1,0,0,0,0,0,0)
$$

\subsection{Two time scale simulations}

\subsubsection{Academic example}

In this section, we aim at investigating the time scale separation properties of system (6), or equivalently of system $(1,2)$. All parameters values are taken from Table 2 except for the infection and recovery rates, $\beta_{P}=\frac{\beta_{P}^{\prime}}{\varepsilon}, \beta_{T}=\frac{\beta_{T}^{\prime}}{\varepsilon}$, $\gamma=\frac{\gamma^{\prime}}{\varepsilon}$. They are chosen such that $N_{T} \beta_{T}^{\prime}, N_{P} \beta_{P}^{\prime}$, and $\gamma^{\prime}$ have the same order of magnitude as the remaining parameters, where $N_{T}$ and $N_{P}$ are typical population sizes for the transiently and persistently infected animals respectively. Equilibrium state (17) gives an indication about these typical sizes. $\beta_{P}^{\prime}=0.5$, $\beta_{T}^{\prime}=0.1$, and $\gamma^{\prime}=1$ are adequate values.

In order to illustrate the effect of time scale separation, we simulate and compare reduced system (9) with complete system (6) using different values of $\varepsilon$ $(\varepsilon \in\{1,1 / 3,1 / 10,1 / 100\})$. To compare those systems, subscript $r$ is used for the variables corresponding to the reduced system plus the quasi steady state; 
for example $W_{r}=\left(X_{r}, Z^{*}\right)$, where $X_{r}$ is the solution of (9) and $Z^{*}$ is given by (8). Let us also note $W^{*}=\left(X^{*}, Z^{*}\right)$. In the simulations, centred variables are represented. They are obtained by shifting the original variables of their equilibrium values, i.e. $W-\bar{W}(\varepsilon)$ for the complete model and $W_{r}-W^{*}$ for the reduced model.

Fig. 3 represents the total population size obtained over a 80-year period. We note a static error between the complete and reduced systems in Fig. 3(a), which decreases as $\varepsilon$ diminishes: from 16 individuals for $\varepsilon=1$ to less than 2 individuals for $\varepsilon=1 / 100$. This error is due to the $\varepsilon$-dependency of the complete system steady state $\bar{W}(\varepsilon)$. The equilibrium of the reduced model $W^{*}$ corresponds to $\varepsilon=0$. To bypass this static error effect we look at the centred variables in Fig. 3(b), which all tend towards zero. However, we notice that the transient dynamics approximation is then deteriorated.

Fig. 4 represents the centred infected population size, consisting of all transiently and persistently infected individuals, over a 4-year period. At the beginning of the simulations, a transient error appears between the complete and reduced systems, because of the neglected fast dynamics. It lasts longer for higher values of $\varepsilon$ : up to 3 years for $\varepsilon=1$, but less than 6 months for $\varepsilon=1 / 10$ and barely noticeable for $\varepsilon=1 / 100$. From these simulations, we deduce that with $\varepsilon=1 / 10$, we obtain a decent approximation of the complete system by the reduced system in the transient phase.

Consequently, to approximate the original system (1), we use a "shifted" reduced system to compensate for the static error; "shifted" variables correspond to centred variables with an additional $\varepsilon$-dependent offset corresponding to the equilibrium of the original system: $W_{r}-W^{*}+\bar{W}(\varepsilon)$. With $\varepsilon$ values lower 


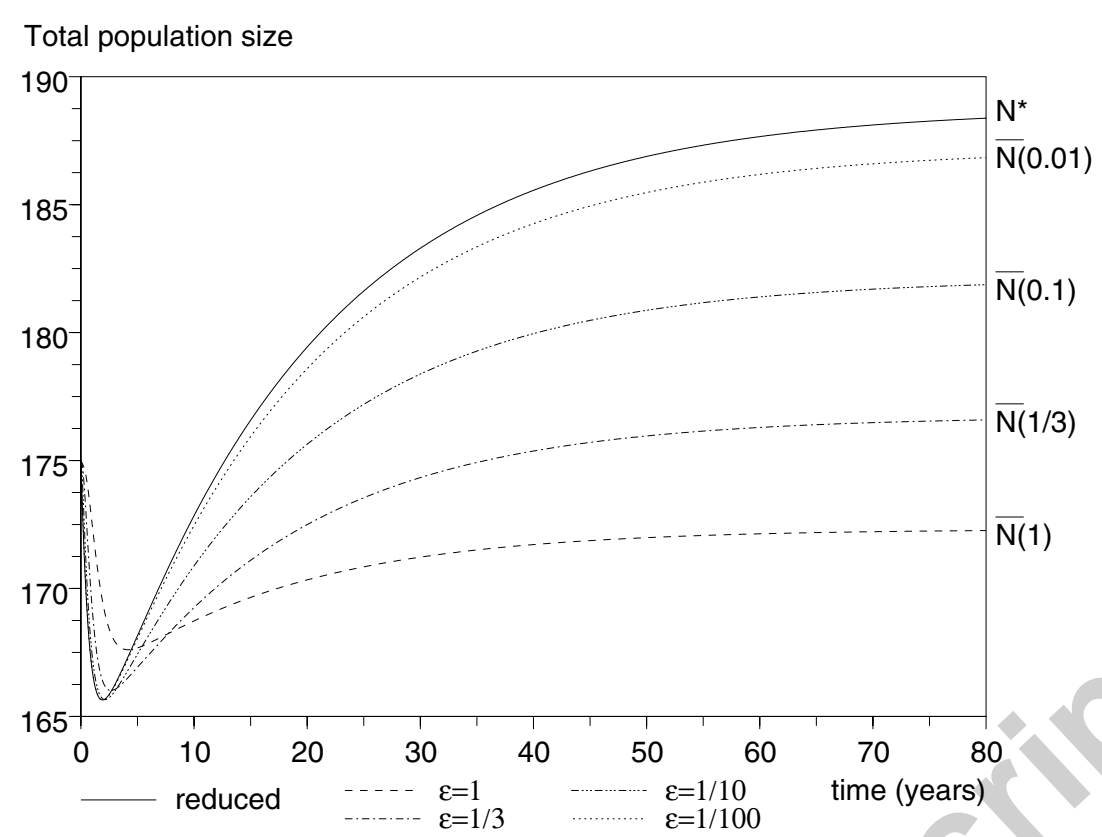

(a) Non centred

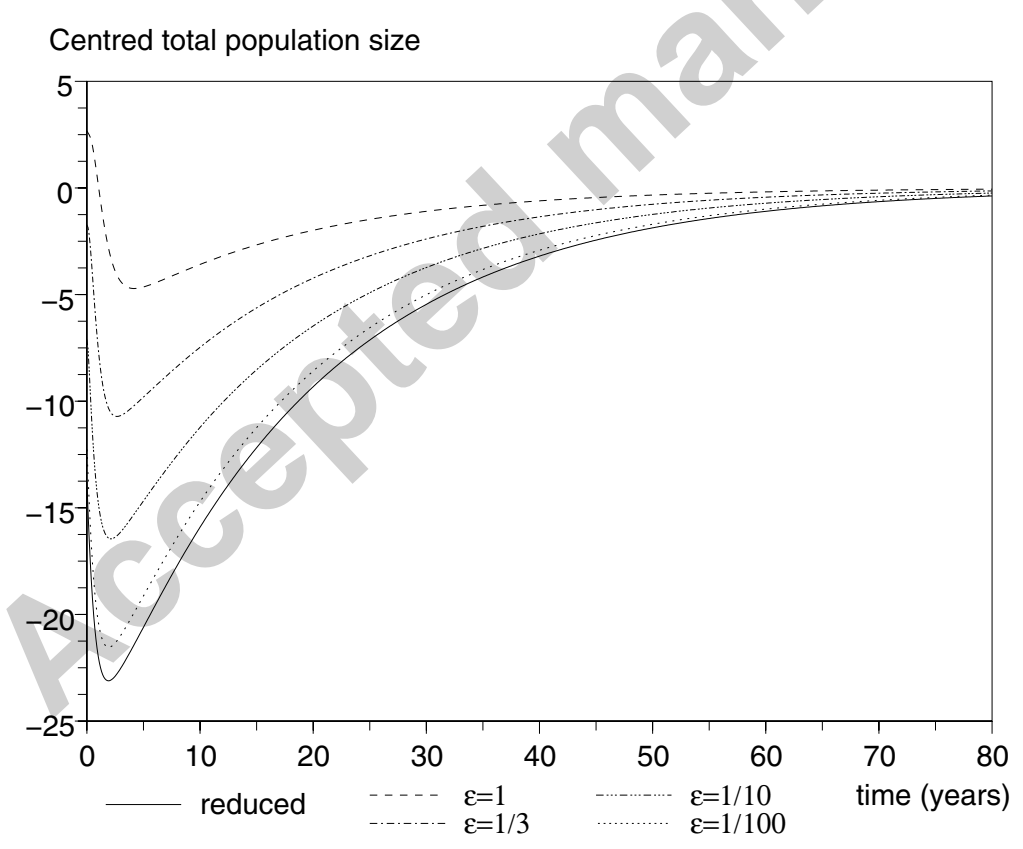

(b) Centred

Fig. 3. Total population size as a function of time for the complete system (6) for $\varepsilon \in\{1,1 / 3,1 / 10,1 / 100\}$ and the reduced system (9). The centred graphs correspond to the total population size $N=A_{1}+A_{2}+P_{1}+P_{2}+R_{2}$ shifted of its equilibrium value, i.e. $N-\bar{N}(\varepsilon)$ for the complete system and $N_{r}-N^{*}$ for the reduced system. $\beta_{P}^{\prime}=0.5, \beta_{T}^{\prime}=0.1$, and $\gamma^{\prime}=1$; the remaining parameters are given in Table 2. 


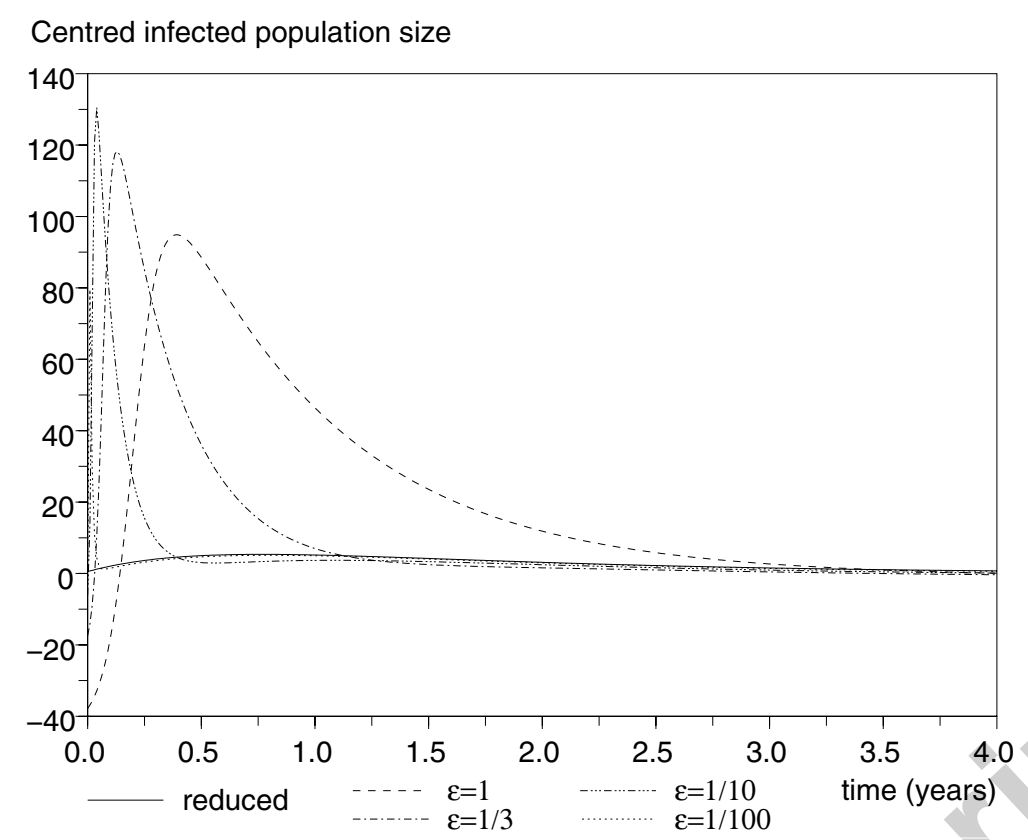

Fig. 4. Centred infected population size as a function of time for the complete system (6) for $\varepsilon \in\{1,1 / 3,1 / 10,1 / 100\}$ and the reduced system (9). The graphs correspond to the infected population size $I=T_{1}+T_{2}+P_{1}+P_{2}$ shifted of its equilibrium value, i.e. $I-\bar{I}(\varepsilon)$ for the complete system and $I_{r}-I^{*}$ for the reduced system. $\beta_{P}^{\prime}=0.5, \beta_{T}^{\prime}=0.1$, and $\gamma^{\prime}=1$; the remaining parameters are given in Table 2.

than $1 / 10$, we hence obtain a satisfying trade-off between the asymptotic and transient dynamics for the approximation.

\subsubsection{Biological example}

We use here the set of biologically relevant values given in Table 2. According to the academic example shown above, with a time scale separation corresponding to $\varepsilon \leqslant 1 / 10$, the reduced model is a decent approximation of the complete system. We therefore set $\varepsilon=1 / 10$ and check if the assumptions on the order of magnitude of the parameters are fulfilled (see section 2.2). 
From the endemic equilibrium values shown in (17), $N_{P} \simeq 1$ and $N_{T} \simeq 2$ are typical population sizes for the persistently and transiently infected animals respectively. Hence $\beta_{T}^{\prime} N_{T}, \beta_{P}^{\prime} N_{P}$ and $\gamma^{\prime}$ should at least have the same order of magnitude as the remaining parameters.

With $\varepsilon=1 / 10$, we obtain the following values for the infection and recovery rates: $\beta_{T}^{\prime}=6 \times 10^{-3}, \beta_{P}^{\prime}=0.1$, and $\gamma^{\prime}=2.4 . \gamma^{\prime}$ is higher than the remaining parameter, $N_{P} \beta_{P}^{\prime}$ is a little lower, $N_{T} \beta_{T}^{\prime}$ is much lower. This means that the time scale separation between demography and infection is poor, whereas it is relevant between recovery and the other phenomena involved in the model.

This result is shown on Fig. 5, when comparing the original and shifted reduced systems over a 20-year long period. The approximation is rather good on the long run for the infected population, but not as satisfying for the other variables.

\subsection{Three time scale simulations}

In this section, we present numerical simulations for system (12), or equivalently system $(1,3)$. We still use the realistic parameter values in Table 2 and the initial condition (18) to perform these simulations. In order to separate the infection and recovery time scales, still assuming that $\varepsilon=1 / 10$, we need to set $\varepsilon^{\prime}<\varepsilon$ such that the recovery rate $\gamma^{\prime \prime}$ has the same order of magnitude as the remaining parameters; $\varepsilon^{\prime}=1 / 24$ leads to $\gamma^{\prime \prime}=1$, which is a suitable value, since $0<1 / 24<1 / 10$.

We use the previous notations for the reduced system and similarly denote 


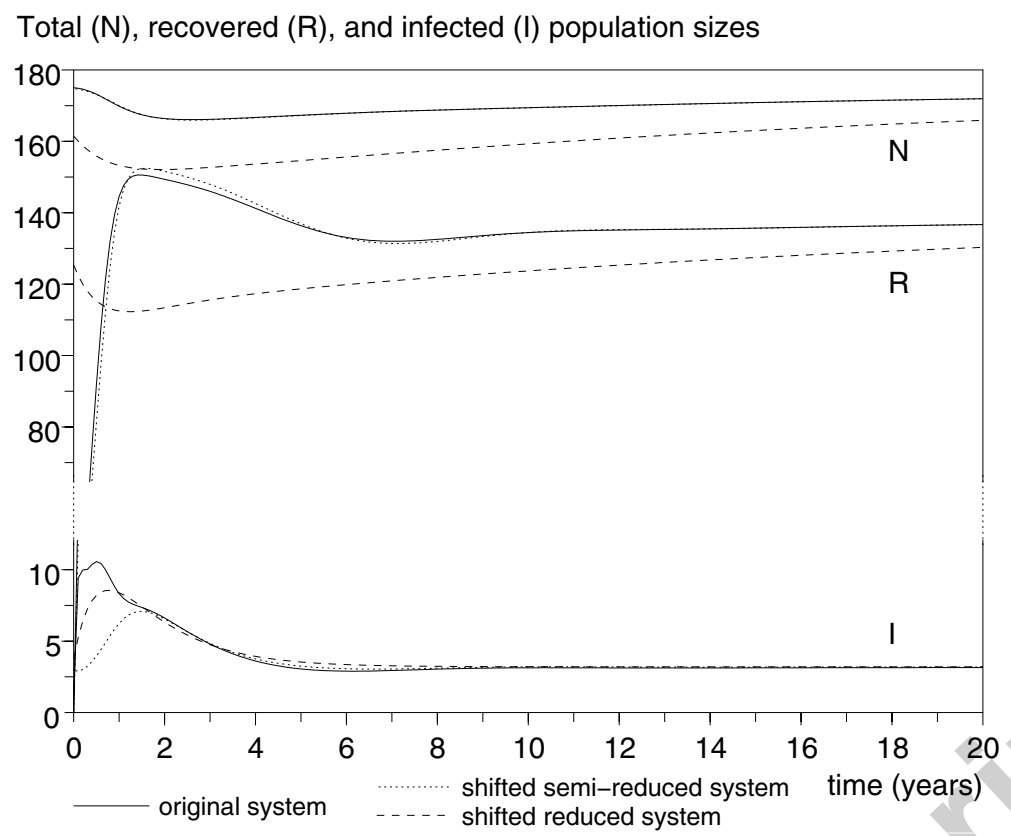

Fig. 5. Total $\left(N=S_{1}+S_{2}+T_{1}+T_{2}+P_{1}+P_{2}+R_{1}+M_{2}+R_{2}\right)$, recovered $\left(R=R_{1}+M_{2}+R_{2}\right)$, and infected $\left(I=T_{1}+T_{2}+P_{1}+P_{2}\right)$ population sizes as functions of time for: [solid lines] original system $(1,3), \bar{W}\left(\varepsilon, \varepsilon^{\prime}\right)$ being its steady state; [dotted lines] shifted semi-reduced system $W_{s}-\widetilde{W}(\varepsilon)+\bar{W}\left(\varepsilon, \varepsilon^{\prime}\right)$, where $W_{s}$ and $\widetilde{W}(\varepsilon)$ are respectively the solution and steady state of (14) plus quasi steady state (13); [dashed lines] shifted reduced system $W_{r}-W^{*}+\bar{W}\left(\varepsilon, \varepsilon^{\prime}\right)$, where $W_{r}$ and $W^{*}$ are similarly defined for (9) plus (8). Parameter values are given in Table $2, \bar{W}$ in $(17), \varepsilon=1 / 10, \varepsilon^{\prime}=1 / 24$.

with subscript $s$ the variables corresponding to the semi-reduced system plus the quasi steady state; for example $W_{s}=\left(X_{s}, \widetilde{Z}(\varepsilon)\right)$, where $X_{s}$ is the solution of $(14)$ and $\widetilde{Z}(\varepsilon)$ is given by (13). Let us also note $\widetilde{W}=(\widetilde{X}, \widetilde{Z})$.

In Fig. 5, we compare the original system (1) with the shifted semi-reduced and reduced systems over a 20-year long period. As previously, the (semi)reduced variables are shifted to compensate for the static error. With two or three time scales, the reduced systems are similar, so we use the same shift for the reduced variables: $W_{r}-W^{*}+\bar{W}\left(\varepsilon, \varepsilon^{\prime}\right)$. The shifted semi-reduced variables 
are similarly defined: $W_{s}-\widetilde{W}(\varepsilon)+\bar{W}\left(\varepsilon, \varepsilon^{\prime}\right) . \bar{W}\left(\varepsilon, \varepsilon^{\prime}\right)$ is the steady state of complete system (12); its numerical value is given in (17).

As expected, the semi-reduced system is a valid approximation of the complete system, in the transient phase and in the long time dynamics. As mentioned previously, the reduced system is not a satisfying approximation. However, it seems to fit the original system better than the semi-reduced system for the infected population. This is actually an artifact due to the representation of the aggregated infected population. If split up into transiently and persistently infected populations, as shown in Fig. 6, the semi-reduced system is a better approximation, especially for the persistently infected population. The transient infection dynamics is fast in both reduced systems, hence the transient error.

Fig. 7 compares the original system with the shifted semi-reduced and reduced systems for the juvenile and adult populations. Due to the fast recovery dynamics, there are almost no infected animals, especially in the adult population. BVDV is maintained in the flock because of the very few PI animals that are born and survive. Moreover, most adults are immunised: they were infected either during their juvenile years or as adults and recovered fast. For the biological parameter values given in Table 2, however, the infection dynamics is not really separated from the demographic dynamics. This explains why a relatively large fraction of juveniles remain in the susceptible state after their birth, which can also be seen in the steady state values given by (17). 


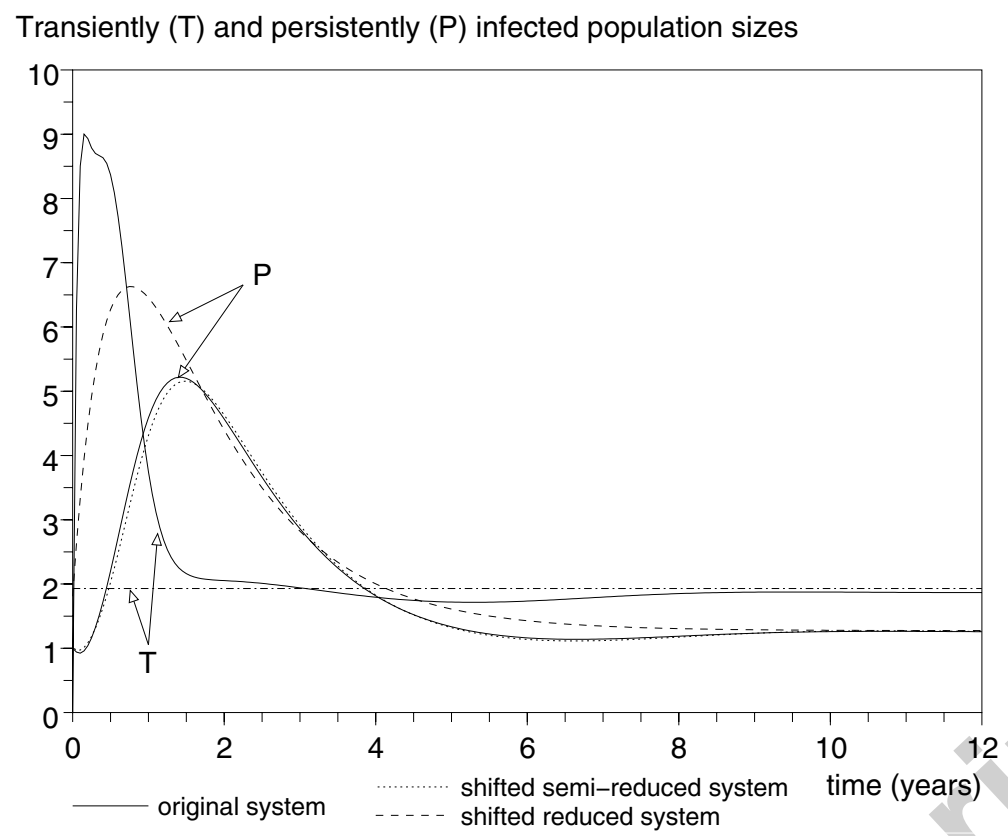

Fig. 6. Transiently $(T)$ and persistently $(P)$ infected population sizes as functions of time: [solid lines] $T$ and $P$ for original system (1,3); [dotted line] shifted $P$ for semi-reduced system $(14,13)$; [dashed line] shifted $P$ for reduced system $(9,8)$; [dash-dotted line] shifted $T$ for the semi-reduced and reduced systems $(T=\bar{T})$. Variable definitions and parameter values are the same as in Fig. 5.

\section{Discussion}

Time scales can be used to reduce the dimension of epidemiological models. Singular perturbations are then valuable tools to corroborate the results of time scale approximations. Here, this method was applied to a model of BVDV spread in a cattle herd.

Even though singular perturbation theory is a well established technique which was already used in previous studies to reduce the dimension of biological systems, our work presents particularly interesting aspects when simultaneously considering theoretical and 


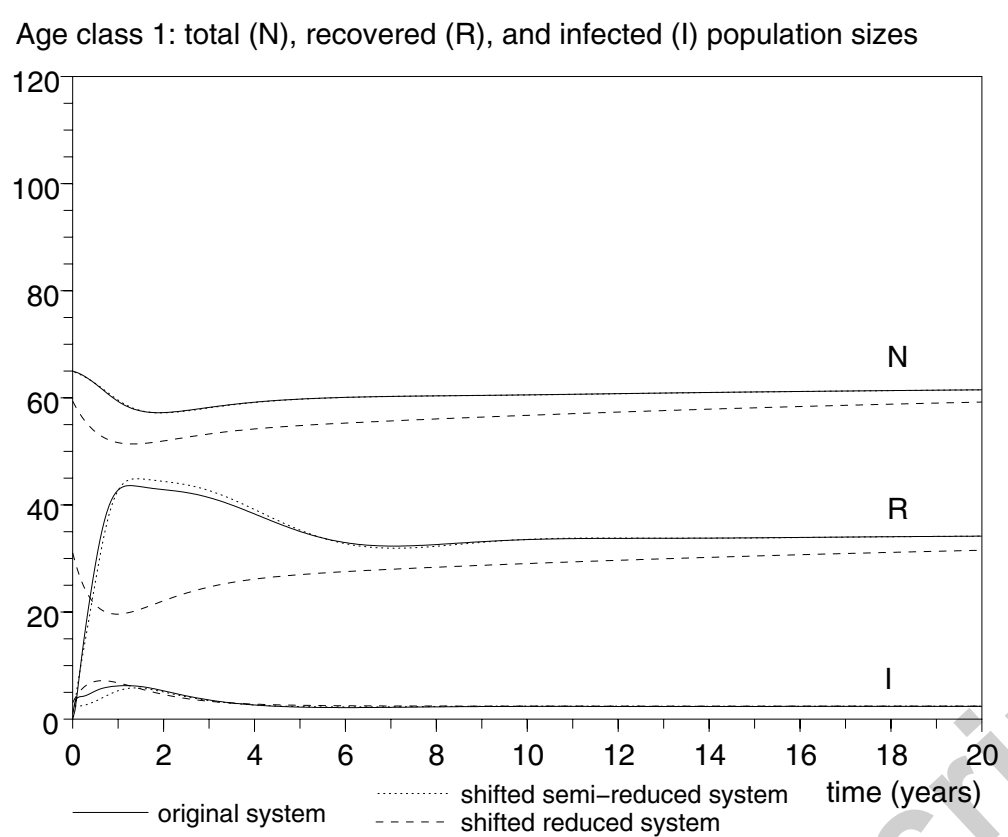

(a) Juveniles

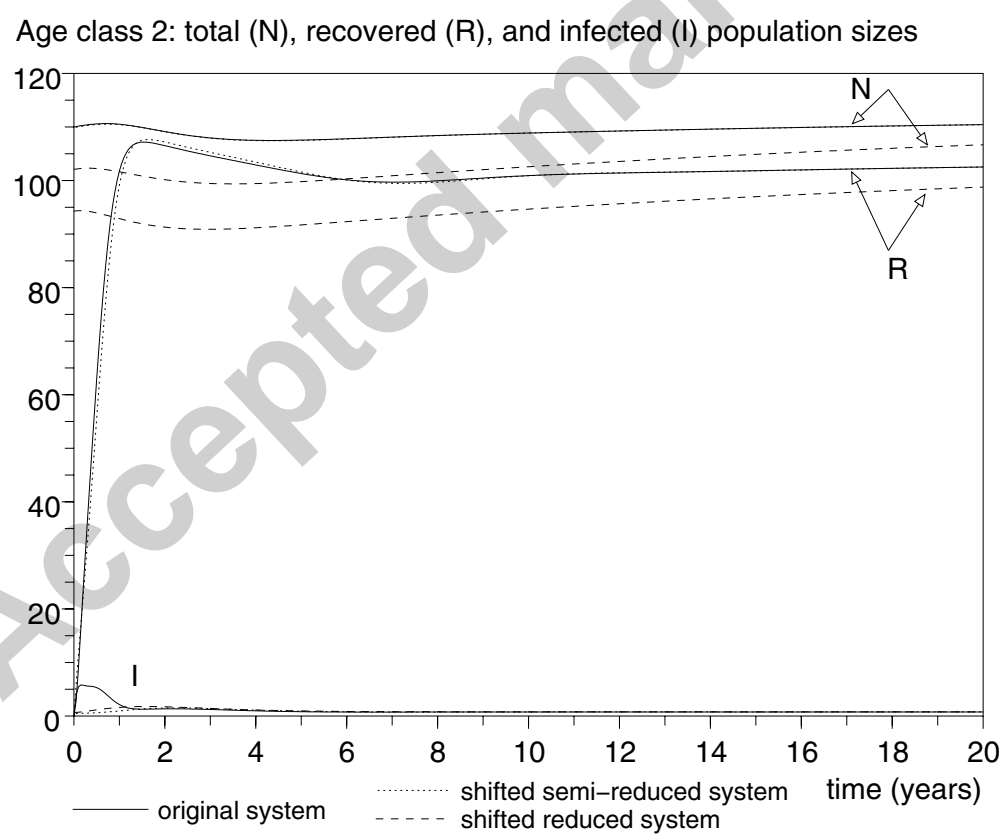

(b) Adults

Fig. 7. Age class specific total, recovered, and infected population sizes as functions of time for: [solid lines] original system $(1,3)$; [dotted lines] shifted semi-reduced system $(14,13) ;$ [dashed lines] shifted reduced system $(9,8)$. Variable definitions and parameter values are the same as in Fig. 33 . 
applied perspectives: (i) contrary to the large majority of similar studies we introduce three time-scales (already used in Lenbury (1996) but in a less disease specific framework) related to biological processes of different speeds leading to satisfying asymptotic approximation of the initial system, obtained without forcing values of biological parameters; (ii) the dimension of our initial model is relatively high compared with other application-oriented studies using singular perturbation tools (such as Song et al. (2002)) and (iii) numerical values of parameters were chosen according to biological relevance, allowing further use of the reduced model in realistic context of BVDV spread in metapopulations.

We have developed a model representing BVDV spread in the herd which captures the main characteristics of the infection and of the host population. The population is structured into two age classes and takes into account the two types of infectious individuals. Firstly, transiently infected animals shed a low amount of virus during a few days (transmission rate $\beta_{T}$ ). Secondly, persistently infected animals may be born after the infection of their dam during mid-pregnancy, and they shed a large amount of virus for their entire life (transmission rate $\beta_{P}$ ). Disease transmission mainly occurs via animal in the herd, but herds can also be contaminated in the field by contact with infected neighbouring herds, especially in endemic regions. Therefore, a specific transmission rate $\left(\beta_{v}\right)$ was considered to account for a constant risk of introducing the pathogen in the herd. As these three transmission rates, $\beta_{T}, \beta_{P}$ and $\beta_{v}$, have different orders of magnitude, and moreover as infection processes go relatively faster than demography, several time scales were identified and used to propose a reduced system. Our model 
of BVDV spread in a herd is still fairly simplified. Cattle herds usually have a finer structure and animals may have less contact with animals from other groups than from their own group (Ezanno et al., 2007). Furthermore, cattle herds are small populations and stochastic models may be more appropriate to represent the spread of a pathogen in such a population. However, the simplified model studied here has a dynamical infection behaviour which is consistent with the one obtained by simulating more detailed models. Therefore, it allows us to use the time scale separation to reduce the herd model and propose a tractable model for further work in a metapopulation context, through the coupling of several hence reduced local models.

In theory, singular perturbation order reduction requires a clear separation between the system time scales to obtain a valid approximation. In practical applications, it can be tested as soon as the separation factor $\varepsilon$ is not more than $\frac{1}{10}$. In our model, a clear time scale separation is achieved for the recovery process. We hence rigorously proved a biological intuition: transiently infected individuals can be neglected when capturing the global dynamics of BVDV spread. However, time scale separation for the infection process is less obvious, at least with our realistic parameter values which lead to very small typical infected population sizes (1-3 infected individuals). In more contaminated herd, the time scale separation and hence the approximation would be better. Simulations showed that even assuming a relatively good time scale separation between demography and infection, there was still a bias between the complete and reduced models. This is due to the $\varepsilon$-dependence of the steady state of the complete model, when the reduced system is computed assuming $\varepsilon=0$ which makes it $\varepsilon$-independent. Using a shifted reduced system compensated for this static error, but it dete- 
riorated the transient phase approximation. The singular perturbation theory allows this drawback to be overcome by the computation of an $\varepsilon$-dependent reduced model, as indicated in Khalil (1996). This should help making the complete reduction to five states more relevant even when the infection force is low. Our next efforts will be concentrated in this direction.

Our study has illustrated that singular perturbations are a very interesting tool to reduce model complexity. It allows to investigate rigorously intuitive simplifications that specialists in a field make on their systems, and to build reduced (though approximated) models that can be used, after coupling with other similar models, in a metapopulation framework.

\section{A Existence of an endemic steady state}

\section{A.1 Original system}

We want to prove the existence of a non negative equilibrium for the original model (1) denoted by vector:

$$
\bar{W}=\left(\overline{S_{1}}, \overline{S_{2}}, \overline{T_{1}}, \overline{T_{2}}, \overline{P_{1}}, \overline{P_{2}}, \overline{R_{1}}, \overline{M_{2}}, \overline{R_{2}}\right),
$$

with $\overline{T_{1}}+\overline{T_{2}}+\overline{P_{1}}+\overline{P_{2}}>0$ so as to obtain an endemic steady state, for a non trivial set of parameters.

We first do some algebra and after successive substitutions and manipulations of different equations of system $(1,2)$, we find that $\overline{M_{2}}, \overline{P_{1}}$ and $\overline{P_{2}}$ are expressed as linear functions of $\overline{T_{2}}, \overline{R_{1}}$ as a linear function of $\overline{T_{1}}$ and $\overline{S_{1}}, \overline{S_{2}}$ and $\overline{R_{2}}$ depend 
linearly on both $\overline{T_{1}}$ and $\overline{T_{2}}$. More precisely, we have:

$$
\begin{gathered}
\overline{M_{2}}=\frac{\gamma^{\prime} \overline{T_{2}}}{\varepsilon\left(\eta+\mu_{2}\right)}, \quad \overline{P_{1}}=\frac{\rho b_{M} \mu_{P_{2}} \gamma^{\prime} \overline{T_{2}}}{\varepsilon c_{P}\left(\eta+\mu_{2}\right)}, \quad \overline{P_{2}}=\frac{\tau \rho b_{M} \gamma^{\prime} \overline{T_{2}}}{\varepsilon c_{P}\left(\eta+\mu_{2}\right)}, \\
\overline{R_{1}}=\frac{\gamma^{\prime} \overline{T_{1}}}{\varepsilon\left(\tau+\mu_{1}\right)}, \quad \overline{R_{2}}=\frac{\tau \gamma^{\prime} \overline{T_{1}}}{\varepsilon\left(\tau+\mu_{1}\right) \mu_{2}}+\frac{\eta \gamma^{\prime} \overline{T_{2}}}{\varepsilon\left(\eta+\mu_{2}\right) \mu_{2}}+\frac{\Delta_{R}}{\mu_{2}}, \\
\overline{S_{1}}=-\left(1+\frac{s_{11}}{\varepsilon}\right) \overline{T_{1}}-\frac{s_{12} \overline{T_{2}}}{\varepsilon}+s_{10}, \quad \overline{S_{2}}=-\frac{s_{21} \overline{T_{1}}}{\varepsilon}-\left(1+\frac{s_{22}}{\varepsilon}\right) \overline{T_{2}}+s_{20},
\end{gathered}
$$

where $s_{11}, s_{12}, s_{10}, s_{21}, s_{22}$ and $s_{20}$ are positive expressions of model parameters.

$\overline{T_{1}}$ (and $\overline{T_{2}}$, their relationship being quasi-symmetric) can be calculated as a root of a third order polynomial $Q_{T_{1}}(x)$ (or $Q_{T_{2}}(x)$ respectively) and has non analytically tractable formula. In order to circumvent this problem, we use a second-order Taylor expansion of $Q_{T_{1}}(x)$ (and $Q_{T_{2}}(x)$ respectively) around $\varepsilon$ and obtain that $\overline{T_{1}} \sim \varepsilon \alpha_{T_{1}}$ (and $\overline{T_{2}} \sim \varepsilon \alpha_{T_{2}}$ respectively), with $\alpha_{T_{1}}>0$ (and $\alpha_{T_{2}}>0$ respectively) under the assumptions $c_{S}>0(4)$ and $c_{P}>0(5)$.

Hence, with these assumptions and if $\varepsilon$ is "small enough", $\overline{T_{1}}$ and $\overline{T_{2}}$ are positive and we can easily see from (A.1) that the positivity is then also verified for $\overline{P_{1}}, \overline{P_{2}}, \overline{R_{1}}, \overline{R_{2}}$ and $\overline{M_{2}}$. Steady states $\overline{S_{1}}$ and $\overline{S_{2}}$ are also positive. Indeed, $\overline{S_{1}}$ can be directly computed from the equation describing the $T_{1}$ dynamic in the complete system (1) as a combination of positive quantities. $\overline{S_{2}}$ is linearly related to $\overline{S_{1}}$, through a positive coefficient, from the equation describing the $S_{2}$ dynamic in system (1).

Consequently, parameter conditions (4-5) and a "small enough" $\varepsilon$ ensure that system $(1,2)$ has a positive endemic steady state. 


\section{A.2 Semi-reduced system}

In the same way, one can prove existence of a unique endemic steady state $\left(\widetilde{A_{1}}, \widetilde{A_{2}}, \widetilde{P_{1}}, \widetilde{P_{2}}, \widetilde{R_{2}}, \widetilde{G_{1}}, \widetilde{G_{2}}\right)$ for the semi-reduced model (14) under similar assumptions. After successive substitutions and manipulations, we obtain the following relations:

$$
\begin{gathered}
\widetilde{R_{2}}=\frac{\tau \widetilde{G_{1}}}{\mu_{2}}+\frac{\eta \widetilde{G_{2}}}{\mu_{2}}+\frac{\Delta_{R}}{\mu_{2}}, \quad \widetilde{P_{1}}=\frac{\rho b_{M} \mu_{P_{2}} \widetilde{G_{2}}}{c_{P}}, \quad \widetilde{P_{2}}=\frac{\tau \rho b_{M} \widetilde{G_{2}}}{c_{P}} \\
\widetilde{A_{1}}=-\frac{\mu_{2}\left((1-\rho) b_{M}-b\right)}{c_{S}} \widetilde{G_{2}}+\frac{\Delta_{S}+\Delta_{R}}{c_{S}} \\
\widetilde{A_{2}}=\frac{\mu_{2} \tau\left((1-\rho) b_{M}-b\right)-\eta c_{S}}{\mu_{2} c_{S}} \widetilde{G_{2}}-\frac{\tau}{\mu_{2}} \widetilde{G_{1}}+\frac{\tau \Delta_{S} \mu_{2}+\tau b \Delta_{R}+\mu_{1} \Delta_{S} \mu_{2}}{\mu_{2} c_{S}}
\end{gathered}
$$

where all parameters are independent of $\varepsilon$.

Then, $\widetilde{G_{1}}$ (or $\widetilde{G_{2}}$ ) can be calculated as a root of a third order polynomial. Using the arguments given above, we obtain the existence of a unique positive solution $\left(\widetilde{G_{1}}, \widetilde{G_{2}}\right)$ under the assumptions $c_{S}>0(4), c_{P}>0(5)$ and for $\varepsilon$

"small enough". Consequently, we obtain the positivity of $\left(\widetilde{P_{1}}, \widetilde{P_{2}}, \widetilde{R_{2}}\right)$ from (A.2); positivity of $\left(\widetilde{A_{1}}, \widetilde{A_{2}}\right)$ can be proved using the equation of system (14) at the equilibrium.

\section{B Singular perturbation theory}

Consider the standard singular perturbation model:

$$
\begin{aligned}
\dot{x} & =f(t, x, z, \varepsilon), \quad x \in \mathbb{R}^{n}, \quad x\left(t_{0}\right)=\xi(\varepsilon), \\
\varepsilon \dot{z}=g(t, x, z, \varepsilon), \quad z \in \mathbb{R}^{m}, & z\left(t_{0}\right)=\phi(\varepsilon) .
\end{aligned}
$$


Call $z^{*}=h(t, x)$ the quasi steady state of equation B.1b with $\varepsilon=0$, i.e. the solution of:

$$
0=g(t, x, z, 0)
$$

The reduced model is:

$$
\dot{x}=f(t, x, h(t, x), 0) \text {. }
$$

Taking $y=z-h(t, x)$, we define the boundary-layer system as:

$$
\frac{d y}{d \nu}=g(t, x, y+h(t, x), 0)
$$

The singular perturbation theory provides the following results (Khalil, 1996).

Theorem 1 Assume that the following conditions are satisfied for all

$$
(t, x, z-h(t, x), \varepsilon) \in[0, \infty) \times B_{r} \times B_{\rho} \times\left[0, \varepsilon_{0}\right] .
$$

- The functions $f, g$ and their partial derivatives with respect to $(x, z, \varepsilon)$ are continuous and bounded.

The function $h(t, x)$ and the Jacobian $[\partial g(t, x, z, \varepsilon) / \partial z]$ have bounded first partial derivatives with respect to their arguments.

The Jacobian $[\partial f(t, x, h(t, x), 0) / \partial x]$ has bounded first partial derivatives with respect to $x$.

The initial data $\xi(\varepsilon)$ and $\phi(\varepsilon)$ are smooth functions of $\varepsilon$.

- The origin of the reduced model (B.2) is exponentially stable.

- The origin of the boundary-layer system (B.3) is exponentially stable uniformly in $(t, x)$.

Then, there exist positive constants $\mu_{1}, \mu_{2}$ and $\varepsilon^{*}$ such that for all

$$
\|\xi(0)\|<\mu_{1}, \quad\left\|\eta(0)-h\left(t_{0}, \xi(0)\right)\right\|<\mu_{2}, \quad \text { and } \quad 0<\varepsilon<\varepsilon^{*}
$$


the singular perturbation problem (B.1) has a unique solution $x(t, \varepsilon), z(t, \varepsilon)$ defined for all $t \geqslant t_{0} \geqslant 0$, and

$$
\begin{gathered}
x(t, \varepsilon)-x^{*}(t)=O(\varepsilon) \\
z(t, \varepsilon)-h\left(t, x^{*}(t)\right)-\hat{y}(t / \varepsilon)=O(\varepsilon)
\end{gathered}
$$

hold uniformly for $t \in\left[t_{0}, \infty\right)$, where $x^{*}(t)$ and $\hat{y}(t)$ are the solutions of the reduced (B.2) and boundary-layer problems (B.3).

Moreover, given any $t_{b}>t_{0}$, there is $\varepsilon^{* *} \leqslant \varepsilon^{*}$ such that

$$
z(t, \varepsilon)-h\left(t, x^{*}(t)\right)=O(\varepsilon)
$$

holds uniformly for $t \in\left[t_{b}, \infty\right)$ whenever $\varepsilon<\varepsilon^{* *}$.

Theorem 2 Assume that the following assumptions are satisfied for all

$$
(t, x, \varepsilon) \in[0, \infty) \times B_{r} \times\left[0, \varepsilon_{0}\right] .
$$

- $f(t, 0,0, \varepsilon)=g(t, 0,0, \varepsilon)=0$.

- The equation $0=g(t, x, z, 0)$ has an isolated root $z=h(t, x)$ such that $h(t, 0)=0$.

- The functions $f, g$ and $h$ and their partial derivatives up to order 2 are bounded for $z-h(t, x) \in B_{\rho}$.

- The origin of the reduced model (B.2) is exponentially stable.

- The origin of the boundary-layer system (B.3) is exponentially stable uniformly in $(t, x)$.

Then, there exists $\varepsilon^{*}>0$ such that for all $\varepsilon<\varepsilon^{*}$, the origin of (B.1) is exponentially stable. 
Note 1 Consider the alternative singular perturbation model:

$$
\begin{aligned}
& \dot{X}=F(t, X, Z, \varepsilon), \quad X \in \mathbb{R}^{n}, \quad X\left(t_{0}\right)=\Xi(\varepsilon), \\
& \varepsilon \dot{Z}=G(t, X, Z, \varepsilon), \quad Z \in \mathbb{R}^{m}, \quad Z\left(t_{0}\right)=\Phi(\varepsilon),
\end{aligned}
$$

where $(\bar{X}(\varepsilon), \bar{Z}(\varepsilon))$ is a non trivial equilibrium of this system.

Call $Z^{*}=H(t, X)$ the quasi steady state, solution of $G(t, X, Z, 0)=0$. The reduced model is:

$$
\dot{X}=f(t, X, H(t, X), 0)
$$

Note $X=\bar{X}(\varepsilon)+x, Z=\bar{Z}(\varepsilon)+z$, and define:

$$
\begin{array}{rlrl}
f(t, x, z, \varepsilon) & =F(t, X, Z, \varepsilon), & & \xi(\varepsilon)=\Xi(\varepsilon)-\bar{X}(\varepsilon), \\
g(t, x, z, \varepsilon) & =G(t, X, Z, \varepsilon), & \phi(\varepsilon)=\Phi(\varepsilon)-\bar{Z}(\varepsilon), \\
h(t, x) & =H(t, X)-Z^{*}, &
\end{array}
$$

As long as $\bar{X}(0)=X^{*}$ and $\bar{Z}(0)=Z^{*}$ exist, with $X^{*}$ the equilibrium of the reduced model, theorems 1 and 2 can be either applied to $(f, g, h)$ or to $(F, G, H)$, using $X^{*}$ for the reduced model and $(\bar{X}(\varepsilon), \bar{Z}(\varepsilon))$ for the system instead of the origin.

\section{Acknowledgements}

This work was carried out with the financial support of the "ANR - Agence Nationale de la Recherche - The French National Research Agency" under the "Programme Agriculture et Développement Durable", project "ANR-05PADD-014, Action collective pour une maîtrise durable de la santé animale: qualification sanitaire en élevage de ruminants (ACDUQ)". 


\section{References}

Arino, O., Sanchez, E., Bravo de la Parra, R., Auger, P., 1999. A singular perturbation in an age-structured population models. SIAM J. Appl. Math. $60(2), 408-436$.

Auger, P., Bravo de la Parra, R., 2000. Methods of aggregation of variables in population dynamics. C. R. Acad. Sci. III 323 (8), 665-674.

Auger, P., Poggiale, J.-C., 1998. Aggregation and emergence in systems of ordinary differential equations. Math. Comput. Modelling 27 (4), 1-21.

Auger, P., Roussarie, R., 1994. Complex ecological models with simple dynamics: From individuals to populations. Acta Biotheoretica 42 (2-3), 111-136.

Baker, J. C., 1987. Bovine viral diarrhoea virus: a review. J. Am. Vet. Med. Assoc. 190 (11), 1449-1458.

Bravo de la Parra, R., Arino, O., Sanchez, E., Auger, P., 2000. A model of an age-structured with two time scales. Math. Comput. Modelling 31 (4-5), $17-26$.

Bravo de la Parra, R., Auger, P., Sanchez, E., 1995. Aggregation methods in discrete models. J. Biol. Systems 3 (2), 603-612.

Brownlie, J., Clarke, M. C., Howard, C. J., Pocock, D., 1987. Pathogenesis and epidemiology of bovine virus diarrhoea virus infection of cattle. Ann. Rech. Vet. 18 (2), 157-166.

Ezanno, P., Fourichon, C., Beaudeau, F., Seegers, H., 2006. Between-herd movements of cattle as a tool for evaluating the risk of introducing infected animals. Anim. Res. 55 (3), 189-208.

Ezanno, P., Fourichon, C., Viet, A.-F., Seegers, H., 2007. Sensitivity analysis to identify key parameters in modelling the spread of bovine viral diarrhoea virus in a dairy herd. Prev. Vet. Med. 80 (1), 49-64. 
Fray, M. D., Paton, D., Alenius, S., 2000. The effects of bovine viral diarrhoea virus on cattle reproduction in relation to disease control. Anim. Reprod. Sci. 60 (Sp. Iss.), 615-627.

González-Guzmán, J., Naulin, R., 1994. Analysis of a model of bovine brucellosis using singular perturbations. J. Math. Biol. 33 (2), 211-223.

Hartley, P. E., Richards, M. S., 1988. A study of the transmission of bovine virus diarrhoea virus between and within cattle herds. Acta Vet. Scand Suppl. 84, 164-166.

Houe, H., 1993. Survivorship of animals persistently infected with bovine virus diarrhoea virus (BVDV). Prev. Vet. Med. 15 (4), 275-283.

Houe, H., 1999. Epidemiological features and economical importance of bovine diarrhoea virus (BVDV) infections. Vet. Microbiol. 64 (2-3), 89-107.

Iwasa, Y., Endreasen, V., Levin, S. A., 1987. Aggregation in model ecosystem. I. Perfect aggregation. Ecol. Model. 37 (3-4), 287-302.

Iwasa, Y., Levin, S. A., Endreasen, V., 1989. Aggregation in model ecosystems II. Approximate aggregation. IMA J. Math. Appl. Med. Biol. 6 (1), 1-23.

Khalil, H. K., 1996. Nonlinear systems, 2nd Edition. Prentice Hall.

Lenbury, Y., 1996. Singular perturbation analysis of a model for a predatorprey system invaded by a parasite. Biosystems 39 (3), 251-262.

Lett, C., Auger, P., Bravo de la Parra, R., 2003. Migration frequency and the persistence of host-parasitoid interactions. J. Theor. Biol. 221 (4), 639-654. Lindberg, A., Brownlie, J., Gunn, G. J., Houe, H., Moennig, V., Saatkamp, H. W., Sandvik, T., Valle, P. S., 2006. The control of bovine viral diarrhoea virus in Europe: today and in the future. Rev. Off. Int. Epizoot. 25, 961-979.

Mc Clurkin, A. W., Littledike, E. T., Cutlip, R. C., Frank, G. H., Coria, M. F., Bolin, S. R., 1984. Production of cattle immunotolerant to bovine viral diarrhoea virus. Can. J. Comp. Med. 48 (2), 156-161. 
Sanz, L., Blasco, A., Bravo de la Parra, R., 2003. Approximate reduction of multi-type Galton-Watson processes with two times scales. Math. Mod. Met. Appl. Sci. 13 (4), 491-525.

Sanz, L., Bravo de la Parra, R., 1999. Variables aggregation in a time discrete linear model. Math. Biosci. 157 (1-2), 111-146.

Sanz, L., Bravo de la Parra, R., 2007. Approximate reduction of multiregional models with environmental stochasticity. Math. Biosci. 206 (1), 134-154.

Song, B., Castillo-Chavez, C., Aparicio, J. P., 2002. Tuberculosis models with fast and slow dynamics: the role of close and casual contacts. Math. Biosci. $180(1-2), 187-205$.

Viet, A.-F., Fourichon, C., Seegers, H., 2007. Review and critical discussion of assumptions and modelling options to study the spread of the bovine viral diarrhoea virus (BVDV) within a cattle herd. Epidemiol. Infect. 135 (5), 706-721. 